\title{
The metalloproteinase ADAM10 requires its activity to sustain surface expression
}

\author{
Anke Seifert ${ }^{1}$ - Stefan Düsterhöft ${ }^{1}$. Justyna Wozniak ${ }^{1}$ Chek Z. Koo ${ }^{2} \cdot$ Michael G. Tomlinson $^{2} \cdot$ Elisa Nuti $^{3}$. \\ Armando Rossello $^{3}$. Doretta Cuffaro ${ }^{3} \cdot$ Daniela Yildiz $^{1,4} \cdot$ Andreas Ludwig $^{1,5}$
}

Received: 21 October 2019 / Revised: 5 March 2020 / Accepted: 17 March 2020 / Published online: 5 May 2020

(c) The Author(s) 2020

\begin{abstract}
The metalloproteinase ADAM10 critically contributes to development, inflammation, and cancer and can be controlled by endogenous or synthetic inhibitors. Here, we demonstrate for the first time that loss of proteolytic activity of ADAM10 by either inhibition or loss of function mutations induces removal of the protease from the cell surface and the whole cell. This process is temperature dependent, restricted to mature ADAM10, and associated with an increased internalization, lysosomal degradation, and release of mature ADAM10 in extracellular vesicles. Recovery from this depletion requires de novo synthesis. Functionally, this is reflected by loss and recovery of ADAM10 substrate shedding. Finally, ADAM10 inhibition in mice reduces systemic ADAM10 levels in different tissues. Thus, ADAM10 activity is critically required for its surface expression in vitro and in vivo. These findings are crucial for development of therapeutic ADAM10 inhibition strategies and may showcase a novel, physiologically relevant mechanism of protease removal due to activity loss.
\end{abstract}

Keywords Metalloproteinase $\cdot$ ADAM10 $\cdot$ Extracellular vesicles $\cdot$ Shedding $\cdot$ Inflammation

$\begin{array}{ll}\text { Abbreviations } \\ \text { ADAM } & \text { A disintegrin and metalloproteinase } \\ \text { AP } & \text { Alkaline phosphatase } \\ \text { BMDM } & \text { Bone marrow-derived macrophage } \\ \text { BTC } & \text { Betacellulin } \\ \text { CHX } & \text { Cycloheximide } \\ \text { ctrl } & \text { Control } \\ \text { DMEM } & \text { Dulbecco's Modified Eagle's Medium }\end{array}$

Electronic supplementary material The online version of this article (https://doi.org/10.1007/s00018-020-03507-w) contains supplementary material, which is available to authorized users.

Andreas Ludwig

aludwig@ukaachen.de

1 Institute of Molecular Pharmacology, Medical Faculty, RWTH Aachen University, Aachen, Germany

2 School of Biosciences, University of Birmingham, Birmingham, UK

3 Department of Pharmacy, University of Pisa, Pisa, Italy

4 Institute of Experimental and Clinical Pharmacology and Toxicology, PZMS, ZHMB, Saarland University, Homburg, Germany

5 Institute of Pharmacology and Toxicology, RWTH Aachen University, Pauwelsstr. 30, 52074 Aachen, Germany

$\begin{array}{ll}\text { EGF } & \text { Epidermal growth factor } \\ \text { ERK } & \text { Extracellular signal-regulated kinase } \\ \text { EV } & \text { Extracellular vesicle } \\ \text { FDR } & \text { False discovery rate } \\ \text { GAPDH } & \text { Glyceraldehyde 3-phosphate dehydrogenase } \\ \text { GI } & \text { GI254023X } \\ \text { HSP } & \text { Heat shock protein } \\ \text { i.p. } & \text { Intraperitoneal } \\ \text { shRNA } & \text { Small hairpin RNA } \\ \text { TAPI } & \text { TAPI-1 } \\ \text { TIMP-1 } & \text { Tissue inhibitor of metalloproteinase 1 } \\ \text { WT } & \text { Wild type }\end{array}$

\section{Introduction}

Surface-expressed proteases of the disintegrin and metalloproteinase (ADAM) family function as critical regulators of physiological and pathophysiological processes during development, inflammation, and cancer [1,2]. ADAM10 is one of the most prominent members of this family. ADAM10 is expressed as a type- 1 transmembrane molecule consisting of an N-terminal prodomain, a metalloproteinase domain, a disintegrin domain, a cysteine-rich domain, a transmembrane helix, and finally a cytoplasmic $\mathrm{C}$-terminal region. 
To a large extent, the function of cell surface-expressed ADAM10 has been attributed to its ability to mediate proteolytic shedding of other surface molecules via its metalloproteinase domain.

ADAM10 can cleave almost 100 different substrates and thus plays critical roles in health and disease [1]. The most important ADAM10 substrate may be Notch controlling cell fate in development [3]. By shedding of Notch family members, ADAM10 controls neuronal differentiation, epidermal integrity, and endothelial angiogenesis [4-6]. However, many more cleavage events have been found to depend on ADAM10 [1]. This includes adhesion molecules of the cadherin family, members of the epidermal growth factor family (EGF and betacellulin), low affinity IgE receptor CD23, ephrins, and inflammatory mediators such as transmembrane chemokines (CXCL16 and CX3CL1) [7]. By regulating these substrates, ADAM10 is involved in endothelial permeability regulation, leukocyte migration $[7,8]$, transactivation of cancer cells [9], fibrogenesis, immune responses, and infectious diseases [10,11].

Since ADAM10 fulfills critical functions in inflammatory as well as proliferative pathologies, it is thought that there exist several mechanisms for upregulating or downregulating ADAM10 activity. Following regulation at the transcriptional and translational level, the synthesized protease is converted from a proform into mature ADAM10 by proteolytic removal of the prodomain. On the cell surface interaction with adapter molecules, conformational changes within the cysteine-rich domain or positioning of the ectodomain in relation to the cell membrane can promote binding of the metalloproteinase domain to the cleavage site of the substrate [12]. Moreover, ADAM10 activity on the cell surface may be downregulated by internalization and proteolytic degradation of the protease [13]. Alternatively, ADAM10 itself can undergo shedding or is released in extracellular vesicles $[14,15]$. Finally, endogenous inhibitors such as the tissue inhibitor of metalloproteinases (TIMP)-1 can block the activity of the protease [16].

Synthetic small molecule inhibitors of ADAM10 have been tested for their potential to block inflammatory or proliferative events. Such inhibitors were identified among the group of hydroxamate-based pseudopeptide metalloproteinase inhibitors [17]. These inhibitors bind to the protease via residues of their pseudopeptide backbone and coordinate the zinc ion in the active site of the proteases via their hydroxamate group. In previous studies, we have identified and characterized the reverse hydroxamate inhibitor GI254023X (in the following abbreviated as GI) as potent and preferential inhibitor of ADAM10-mediated shedding events [18]. By measuring the activity of the recombinant metalloproteinase domains of ADAM10 or ADAM17, we showed that the inhibitor has a 100-fold higher potency to block ADAM10 than ADAM17. Structural data indicate that the $\mathrm{S} 1$ binding pocket of ADAM10 possesses an extended cavity, which is absent in ADAM17 [19] and hence, the large phenylpropyl residue of $\mathrm{GI}$ in the $\mathrm{P} 1$ ' position would fit in the S1' pocket of ADAM10 but not in that of ADAM17 [18]. Consistent with these findings, the inhibitor effectively blocks ADAM10-mediated ligand-induced shedding of Notch, constitutive and ionomycin-induced shedding of the low affinity IgE receptor CD23, cadherins (N,E, VE) transmembrane chemokines (CXCL16 and CX3CL1) and growth factors (EGF and betacellulin) [7, 20-26]. Recent studies also showed that the inhibitor can be used to block shedding events in vivo and prevent inflammatory processes and fibrosis development in lungs and kidney, respectively $[10,11]$.

In most previous studies, GI-mediated effects were investigated for only a short period of time which is required for shedding. It is clear that only a few minutes are sufficient for GI to interact with the majority of ADAM10 and that the binding to the active site is likely causing immediate inhibition of ADAM10 activity. However, the long-term treatment effects on ADAM10 over a period of several hours or even days remain unknown. Such knowledge would not only be vital to develop effective ADAM10 inhibition strategies but also allow further important insight in the biology of ADAM10. Here, we studied the long-term effects of GI exposure on surfaceexpressed and total ADAM10. Surprisingly, we observed that GI exposure led to downregulation of mature ADAM10 which was not due to decreased ADAM10 synthesis but coincided with ADAM10 release in extracellular vesicles. This depletion of ADAM10 resulted in long-term suppression of cellular ADAM10 activity. After removal of GI, the loss of ADAM10 surface activity could only be overcome by de novo synthesis of the protease. The depletion of ADAM10 was not restricted to GI or related hydroxamate inhibitors but was also seen with the natural inhibitor TIMP-1, suggesting that this is a physiological phenomenon. Moreover, mutational inactivation of ADAM10 reduced its surface expression and GI could not further downregulate the inactive protease underlining the importance of ADAM10 activity for its surface expression. Finally, intraperitoneal (i.p.) administration of GI in mice also led to sustained ADAM10 downregulation indicating in vivo relevance of this mechanism. To our knowledge, this is the first report showing that activity of a protease is required to maintain full surface expression and this mechanism may have broader pharmacological implications also for other proteases.

\section{Materials and methods}

\section{Antibodies, recombinant proteins, and chemical compounds}

Mouse monoclonal antibodies against the $\mathrm{N}$-terminus of human ADAM10 (MAB1427), human ADAM17 
(MAB9301), human ERK1/2 (MAB1576), mouse IgG1 isotype control (MAB002), mouse IgG2b isotype control (MAB004), rat monoclonal antibody against the N-terminus of murine ADAM10 (MAB946), and rat IgG2a isotype control (MAB006) were from R\&D Systems (Wiesbaden, Germany). Mouse monoclonal antibodies against human/ murine GAPDH (MA5-15738), human CD9 (10626D) and rat monoclonal eFluor 450-conjugated CD11b antibody (480112-82) were from Thermo Fisher Scientific (Waltham, USA). Allophycocyanin (APC)-conjugated goat anti-mouse (115-135-164), phycoerythrin (PE)-conjugated goat anti-rat (112-116-071), horseradish peroxidase (HRP)-conjugated goat anti-mouse, goat anti-rabbit and goat anti-rat antibodies were from Jackson ImmunoResearch Laboratories, Inc. (West Grove, USA). Rabbit polyclonal antibodies against the C-terminus of ADAM17 (AB19027) and ADAM10 (AB19026) were from Merck Millipore (Darmstadt, Germany). Rabbit polyclonal anti-human HSP70 antibody (EXOAB-Hsp70A-1) was from System Biosciences (Palo Alto, USA). Mouse monoclonal antibody to flotillin-1 (610821) was from BD Biosciences (Heidelberg, Germany). FITC-conjugated rat monoclonal anti-mouse F4/80 antibody (MCA497F) was from Serotec (Bio-Rad, Hercules, USA). The metalloproteinase inhibitors GI254023X, MN8, and MN8-Cy5.5 (CAM36) were synthesized and characterized as preferential ADAM10 inhibitors as previously reported [18, 21, 27]. TAPI-1 was from Merck (Darmstadt, Germany). $\mathrm{NH}_{4} \mathrm{Cl}$ and saponin were from Carl Roth (Karlsruhe, Germany), monensin was from Tocris Bioscience (Bristol, UK), cycloheximide, ionomycin, and bafilomycin A1 were from Sigma-Aldrich (St. Louis, USA), ikarugamycin was from biomol (Hamburg, Germany), recombinant human TIMP-1 (970-TM-010) was from R\&D Systems (Minneapolis, USA), E. coli pHrodo (P35366), EZ-Link ${ }^{\mathrm{TM}}$ Sulfo-NHS-LC-Biotin, and Aldehyde/Sulfate latex beads were from Thermo Fisher Scientific (Waltham, USA), Streptavidin-Sepharose High Performance was from GE Healthcare (Chicago, USA) and the ADAM10 substrate peptide (PRYEAYKMG) was from peptides \& elephants (Hennigsdorf, Germany).

\section{Cell culture, transduction, and transfection}

The human monocytic leukemia cell line THP-1 was cultured in RPMI medium with 10\% FCS (PanBiotech, Aidenbach, Germany) and 1\% penicillin-streptomycin (SigmaAldrich, St. Louis, USA). The alveolar lung carcinoma epithelial cell line A549, the human embryonic kidney cell line HEK293, and the murine macrophage cell line RAW264.7 were cultured in Dulbecco's Modified Eagle's Medium (DMEM) with 10\% FCS and 1\% penicillin-streptomycin. Human neutrophils from healthy donors and murine BMDMs were isolated and cultured as described [28]. Experiments with human neutrophils were approved by the local ethical committee of the RWTH Aachen Medical Faculty (EK 259/13) and conform the declaration of Helsinki. ADAM10-deficient HEK293 cells were generated by CRISPR/Cas 9 technique and kindly provided by Björn Rabe, University of Kiel [29].

Short hairpin RNA (shRNA) targeting ADAM10 was inserted into the lentiviral expression vector pLVTHM as described [30]. For expression of shRNA, recombinant lentiviruses were produced as described [30]. For transduction, $2 \times 10^{5}$ THP- 1 cells were seeded into 12 -wells and concentrated lentivirus preparation $(5 \mu \mathrm{l})$ was added. To enhance the transduction efficiency polybrene $(4 \mu \mathrm{g} / \mathrm{ml}$, SigmaAldrich, St. Louis, USA) was used.

For overexpression of murine ADAM10, coding DNA was inserted into the pMOWS system by usage of the NEBuilder HiFi DNA Assembly Cloning Kit from New England BioLabs (Frankfurt, Germany) and transiently transfected into ADAM10-deficient HEK293 cells using Lipofectamine 3000 (Thermo Fisher Scientific, Waltham, USA).To generate the E/A mutant, the GAA codon at position 1376-78 of murine ADAM10 cDNA was replaced by GCA with the CloneAmp HiFi PCR Premix from Takara (Takara Bio Europe, St-Germain-en-Laye, France). To generate the HE switch mutant, the GAA codon at position 1376-78 and the CAT codon at position 1385-87 of murine ADAM10 cDNA were exchanged.

\section{Cell treatment}

For treatment with inhibitors, cells were seeded in fully supplemented medium with GI254023X $(10 \mu \mathrm{M})$, TAPI-1 $(10 \mu \mathrm{M})$ or $0.1 \%$ dimethylsulfoxide (DMSO). If not stated differently, the treatment time was $24 \mathrm{~h}$. To analyze the recovery of ADAM10, cells were washed with PBS and received new complete growth medium for up to $24 \mathrm{~h}$. For analysis of cellular protein content, cell lysates were generated using a lysis buffer containing $20 \mathrm{mM}$ Tris- $\mathrm{HCl}$, $150 \mathrm{mM} \mathrm{NaCl}, 1 \%$ TritonX-100, $5 \mathrm{mM}$ EDTA, $1 \mathrm{mM}$ PMSF, $10 \mathrm{mM}$ 1,10-phenanthroline monohydrate, onefold Complete (Roche) and $10 \mu \mathrm{M}$ GI. After 20 min incubation on ice, lysates were cleared by 10 min centrifugation at $16,100 \times g$ and analyzed via a BCA assay (Interchim, Montluçon, France) according to manufacturer's instructions. For flow cytometric analysis of ADAM10 and ADAM17, surface expression adherent cells were harvested using accutase (Sigma-Aldrich, Steinheim, Germany).

\section{Flow cytometric analysis}

PBS supplemented with $0.2 \%$ BSA was used as assay buffer, and all steps of the staining process were performed at $4{ }^{\circ} \mathrm{C}$. Cells or precipitates of extracellular vesicles and latex beads were stained with primary antibody or appropriate isotype 
control for 45-60 min on ice. Detection of bound antibodies was performed using APC- or PE-conjugated secondary antibody. For permeabilization, cells were fixed with $2 \%$ PFA and afterwards in all steps, $0.1 \%$ saponin was added. The fluorescence signal was analyzed by flow cytometry (LSRFortessa, BD Biosciences, Heidelberg, Germany) and evaluated with FlowJo V10 software. Binding of MN8Cy5.5 to surface-expressed ADAM10 was determined by staining cells with $10 \mu \mathrm{M}$ inhibitor, washing and subsequent flow cytometric analysis. The unspecific signal which was determined by preincubation with $10 \mu \mathrm{M}$ GI as a competitor prior to staining with $10 \mu \mathrm{M}$ MN8-Cy5.5 was subtracted from the obtained fluorescence signal.

\section{Western blotting}

Samples from cell lysates ( $20 \mu \mathrm{g}$ total protein), precipitation of surface proteins or vesicle preparations (see below) were heated in reducing or non-reducing SDS-sample buffer (250 mM Tris $\mathrm{HCl}(\mathrm{pH} 6.8), 50 \%(\mathrm{w} / \mathrm{v})$ glycerol, $10 \%$ (w/v) SDS, $0.1 \%$ bromophenol blue with or without $5 \%$ $\beta$-mercaptoethanol) and subjected to SDS-polyacrylamide gel electrophoresis using 10\% Tris-glycine gels. Proteins were transferred onto polyvinylidene difluoride membranes (Hybond-P, Amersham). Membranes were blocked with 5\% (w/v) non-fat dry milk in PBS with $0.05 \%$ Tween for $1 \mathrm{~h}$ and probed with primary antibodies against ADAM10 $(0.5 \mu \mathrm{g} / \mathrm{ml})$ or marker proteins for $1 \mathrm{~h}$ at room temperature or overnight at $4{ }^{\circ} \mathrm{C}$ followed by incubation with HRP-coupled secondary antibodies (diluted 1:30,000) for $1 \mathrm{~h}$. After addition of enhanced chemiluminescence substrate (ECL Prime, GE Healthcare), signals were recorded using the LAS 3000 Image Analyzer ${ }^{\circledR}$ (Fujifilm, Tokyo, Japan) and quantified using the open source ImageJ software (developed by Wayne Rasband, NIH). ADAM10 migrates faster under non-reducing conditions than under reducing conditions. Therefore, detection by $\mathrm{N}$-terminal ADAM10 antibody which requires non-reducing conditions, results in a $55 \mathrm{kDa}$ band whereas the C-terminal ADAM10 antibody detects mature ADAM10 at $70 \mathrm{kDa}$ under reducing conditions.

\section{Surface biotinylation}

To biotinylate surface proteins, the cells were washed three times with ice-cold PBS and incubated for $30 \mathrm{~min}$ at $4{ }^{\circ} \mathrm{C}$ with EZ-Link Sulfo-NHS-LC-Biotin $(1 \mathrm{mg} / \mathrm{ml}$ in PBS, pH 8.0, Thermo Fisher Scientific, Waltham, USA). The biotin reagent was quenched by washing the cells three times with $100 \mathrm{mM}$ glycine in PBS. Cells were lysed as described above and $90 \%$ of the lysates were incubated overnight with streptavidin-sepharose high performance beads (GE Healthcare) at $4{ }^{\circ} \mathrm{C}$ on a rotary mixer to isolate biotinlabeled proteins. The sepharose beads were spun down and washed three times with lysis buffer. Finally, the beads were resuspended in reducing (C-terminal ADAM10) or nonreducing (N-terminal ADAM10) SDS-sample buffer $(2 \times)$ and analyzed via western blot.

\section{Extracellular vesicle preparation}

Extracellular vesicles were prepared as described previously [31]. $20 \times 10^{6}$ THP- 1 or A549 cells were cultured in serumfree medium for $24 \mathrm{~h}$. Extracellular vesicles were prepared from supernatants by centrifugation for $10 \mathrm{~min}$ at $300 \times g$, followed by $20 \mathrm{~min}$ at $2000 \times \mathrm{g}$ and $30 \mathrm{~min}$ at $10,000 \times g$. The resulting supernatant was filtered through a $0.22 \mu \mathrm{m}$ membrane and extracellular vesicles were sedimented at $100,000 \times g$ for $75 \mathrm{~min}$ at $4{ }^{\circ} \mathrm{C}$ using a Beckman rotor Type Ti70. The sediment was resuspended in a high volume of ice-cold PBS and sedimented again at $100,000 \times g$ for $75 \mathrm{~min}$. For western blot analysis, vesicles were directly dissolved in SDS-sample buffer. For flow cytometric analysis, vesicles were resuspended in PBS and incubated with $50 \mu \mathrm{l}$ aldehyde/ sulphate latex beads ( $4 \% \mathrm{w} / \mathrm{v}, 3.8 \mu \mathrm{m}$ diameter) overnight at $4{ }^{\circ} \mathrm{C}$. Subsequently, the reaction was stopped by addition of $100 \mathrm{mM}$ glycine. After washing twice, bead conjugates were subjected to flow cytometry as described above.

\section{Substrate cleavage}

ADAM10 activity on THP-1 cells was studied in terms of CXCL16 release. $2.5 \times 10^{5}$ THP-1 cells were pretreated for $24 \mathrm{~h}$ with $10 \mu \mathrm{M}$ GI or vehicle, washed twice with PBS, incubated in fresh growth medium for another $24 \mathrm{~h}$ with or without GI and stimulated to express CXCL16 by incubation with E. coli pHrodo (THP-1 to E. coli ratio is 1:5). Conditioned media were cleared by centrifugation ( $5 \mathrm{~min}$, $16,100 \times g, 4{ }^{\circ} \mathrm{C}$ ) and soluble CXCL16 was quantified per ELISA as described [23]. The substrate cleavage assay using betacellulin $\mathrm{N}$-terminally tagged with alkaline phosphatase (AP) has been described previously [32]. HEK293 cells were transfected with plasmid encoding the betacellulinAP fusion protein by usage of Lipofectamine 3000 (Thermo Fisher Scientific, Waltham, USA). $48 \mathrm{~h}$ after transfection, the cells were washed and treated for 45 min with $1 \mu \mathrm{M}$ ionomycin in DMEM without supplements. Subsequently, AP activity was determined over time in the prepared cell lysates and supernatants by adding a $p$-Nitrophenyl phosphate (PNPP) solution (Thermo Fisher Scientific, Waltham, USA). The AP activity was continuously measured at $405 \mathrm{~nm}$ with the FLUOstar Optima. To assess the AP activity, the slope (change of absorption at $405 \mathrm{~nm}$ per min) was calculated. The amount of ADAM10 activity was calculated as PNPP substrate turnover (AP activity) in the supernatant in relation to the total turnover in supernatant plus cell lysate. 


\section{Quantitative PCR analysis}

The mRNA expression level of ADAM10 was measured by quantitative real-time PCR and normalized to the mRNA expression level of glyceraldehyde-3-phosphate dehydrogenase (GAPDH). RNA was extracted using RNeasy Kit (Qiagen, Hilden, Germany) and quantified photometrically (NanoDrop, Peqlab, Erlangen, Germany). $300 \mu \mathrm{g}$ RNA was reverse transcribed using PrimeScript ${ }^{\mathrm{TM}}$ RT Reagent Kit (Takara Bio Europe, St-Germain-en-Laye, France) and PCR reactions were performed using SYBR Premix Ex Taq II (Takara Bio Europe) according to the manufacturer's protocol. The following primers were used: ADAM10 forward, ggattgtggctcattggtgggca; $A D A M 10$ reverse, actctctcggggccgctgac; GAPDH forward, ccagccccagcgtcaaaggtg; GAPDH reverse, cggggctctccagaacatcatcc. All PCR reactions were run on a LightCycler 480 System (Roche, Basel, Schweiz) with 45 cycles of $10 \mathrm{~s}$ denaturation at $95^{\circ} \mathrm{C}$, followed by $30 \mathrm{~s}$ annealing at $61{ }^{\circ} \mathrm{C}(A D A M 10)$, or $66^{\circ} \mathrm{C}(G A P D H)$ and $15 \mathrm{~s}$ amplification at $72{ }^{\circ} \mathrm{C}$. Standard curves were determined using a serially diluted internal standard. Relative quantification was performed with the E-Method from Roche Applied Bioscience using the LightCycler ${ }^{\circledR} 480$ software 1.5 .

\section{Mice}

Animal experiments were approved by the local authorities and performed with 19-21 g male mice (Janvier Labs) on a C57BL/6JRj background (84-02.04.2014.A512; LANUV $\mathrm{NRW}$ ). Mice received a daily intraperitoneal (i.p.) injection of $200 \mathrm{mg} / \mathrm{kg}$ bodyweight GI254023X (300 mM) or DMSO as control over 13 days. Before mice were sacrificed, blood samples were taken retro-orbitally. Lung and liver samples were taken and stored at $-80{ }^{\circ} \mathrm{C}$. For flow cytometric analysis, organ samples were homogenized on ice and erythrocytes were lysed in all samples. Afterwards, cells were stained and subjected to flow cytometry as described above.

\section{Statistics}

Quantitative data are shown as mean and standard deviation (SD) calculated from at least three independent experiments. For a better graphical presentation and comparison flow cytometry, data were normalized by expression in relation to the appropriate controls. Raw data were analyzed by general mixed model analysis (PROC GLIMMIX, SAS 9.4, SAS Institute Inc., Cary, USA) and assumed to be derived from either normal, log normal or beta (percentage data) distributions; residual plots and the Shapiro-Wilk test were used as diagnostics. If necessary, the day of experiment conduction was set as random to assess differences in the size of treatment effects across the results. According to the covtest statement, all data sets were homoscedastic. Multiple comparisons were corrected by false discovery rate (FDR). Significant differences compared to the respective control were indicated as asterisks $(* p<0.05, * * p<0.01, * * * p<0.001)$. In case of additional comparisons, these were specified by bars.

\section{Results}

\section{The metalloproteinase inhibitor GI254023X reduces surface-detectable ADAM10}

Monocytic THP-1 cells were used to study the long-term effects of GI254023X on surface-expressed ADAM10. As demonstrated in detail in previous work, the cells express functional ADAM10 and the protease can be detected on the cell surface by staining with a monoclonal antibody against the extracellular N-terminus of ADAM10 and subsequent flow cytometric analysis [28]. We noted that a 24-h exposure of THP-1 cells to GI resulted in a considerable reduction of surface-detectable GI compared to control cells treated with vehicle (Fig. 1a). In further experiments, we verified that this effect was time- and concentration-dependent reaching about $15 \%$ residual ADAM10 expression after $24 \mathrm{~h}$ treatment with $10 \mu \mathrm{M}$ GI (Fig. 1b, c). Of note, 15 min preincubation, which is enough for optimal inhibition of ADAM10 [21], is not sufficient for downregulation of ADAM10. Moreover, the long-term effect of GI was limited to ADAM10 and not seen for ADAM17 which is not a primary target of the inhibitor (Fig. 1b, d). We then tested TAPI-1 as a potent inhibitor of ADAM17 with additional inhibitory activity on ADAM10 (Fig. 1b, d). This inhibitor also did not affect ADAM17 surface detection. For ADAM10, some downregulation by TAPI- 1 could be observed but not to the same extent as observed by GI treatment (Fig. 1b, d).

We next studied other cell types and found that GI also downregulates surface-detectable ADAM10 in epithelial lung carcinoma A549 cells without affecting ADAM17 (suppl. Fig. 1AB). Similarly, we observed GI-induced reduction of surface-detectable ADAM10 expression in HEK293 cells and primary human neutrophils (suppl. Fig. 1CD). Furthermore, another recently developed ADAM10 inhibitor (MN8) [33] significantly reduced the surface-detectable ADAM10 in THP-1 cells as well (suppl. Fig. 1E). Notably, there was neither an effect of GI on the mRNA expression of ADAM10 in THP-1 cells nor in A549 cells suggesting that ADAM10 seems to be regulated on a posttranslational level rather than on a transcriptional level (suppl. Fig. 1FG).

\section{GI254023X reduces total cellular content of mature ADAM10}

It is either possible that GI reduces the number of ADAM10 molecules on the cell surface or, alternatively, the inhibitor could simply prevent detection of the 

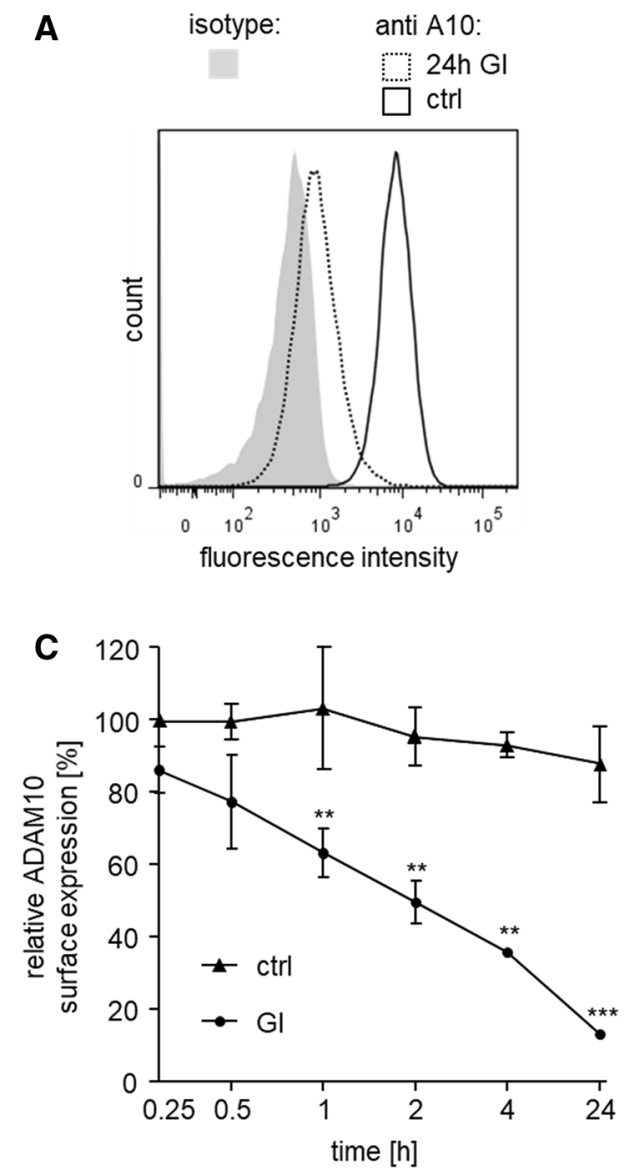

Fig. 1 Effect of metalloproteinase inhibitors GI and TAPI on surface expression of ADAM10 and ADAM17. a THP-1 cells were treated with $10 \mu \mathrm{M}$ GI, or DMSO as vehicle control (ctrl) for $24 \mathrm{~h}$ and subsequently analyzed for surface expression of ADAM10 by flow cytometry. Data are shown as representative histogram. b THP-1 cells were treated with the indicated concentrations of GI, TAPI or vehicle control for $24 \mathrm{~h}$ and subsequently analyzed for surface expression of ADAM10 $(n=3)$. c THP-1 cells were treated with $10 \mu \mathrm{M}$ GI or vehi-

protease. To address this question, ADAM10 expression was studied by western blotting of cell lysates. In general, ADAM10 can be detected by antibodies against the extracellular N-terminus or antibodies against the intracellular $\mathrm{C}$-terminus. The binding sites of these antibodies do not overlap with the protease's active site. In comparison, the small molecule inhibitor GI254023X binds to the active site of ADAM10 and bigger natural inhibitors like TIMP-1 bind the active site and other sites as well (suppl. Fig. 2B) For western blot analysis, we first used the same N-terminal antibody against ADAM10 as for flow cytometry. This antibody detects two close protein bands with only small difference in molecular weight in lysates of THP-1 cells [31]. The specificity of this detection was controlled by shRNA-mediated knockdown of ADAM10 leading to disappearance of both bands (suppl. Fig. 2A). THP-1
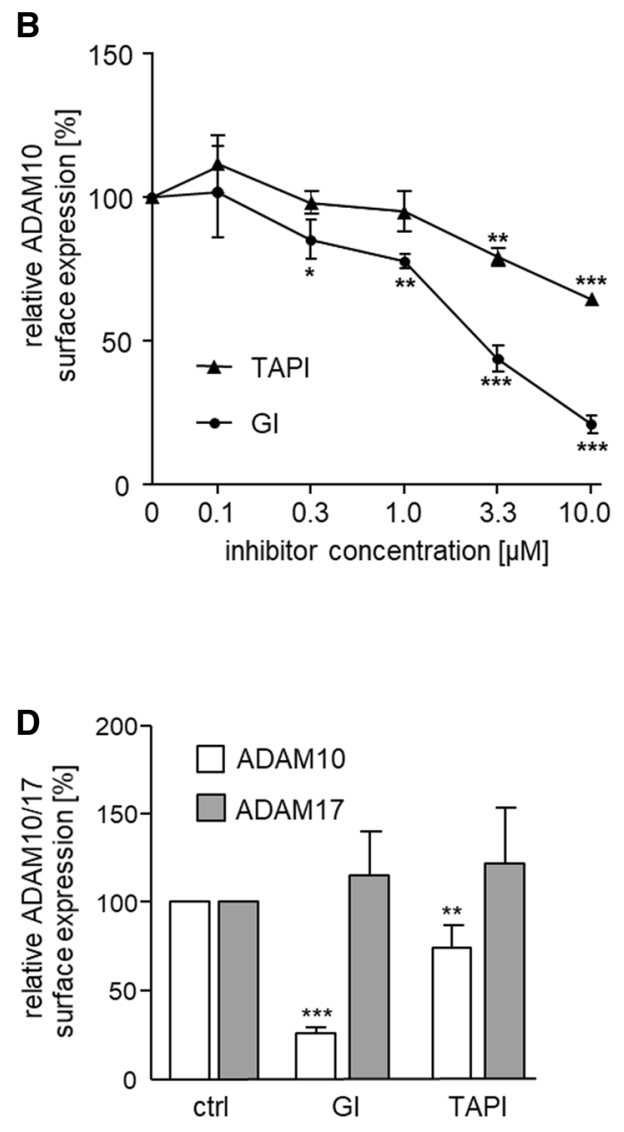

cle control for the indicated periods of time and subsequently analyzed for surface expression of ADAM10 $(n=3)$. d THP-1 cells were treated with $10 \mu \mathrm{M}$ GI, $10 \mu \mathrm{M}$ TAPI or vehicle control for $24 \mathrm{~h}$ and subsequently analyzed for surface expression of ADAM10 $(n=7)$ or ADAM17 $(n=5)$. In b-d, the geometric mean fluorescence of inhibitor-treated cells was calculated in relation to that of the respective control and summarized as mean and SD of at least three independent experiments

cells were then exposed to GI and either lysed or used for biotinylation and precipitation of surface proteins. A clear reduction of ADAM10 protein in the lysate and in the surface precipitate was observed (Fig. 2a, suppl. Fig. 2C). By contrast, treatment with TAPI-1 led to a much weaker reduction of ADAM10 in the THP-1 cell lysate. The findings could be confirmed with A549 cells showing a predominant reduction of ADAM10 in surface precipitates after GI treatment and only a moderate reduction after TAPI-1 treatment (suppl. Fig. 2D).

Since the electrophoresis had to be run under nonreducing conditions for detection by the $\mathrm{N}$-terminal antibody, we could not exclude an influence of remaining inhibitor on the detection of ADAM10. Moreover, it remained unclear whether GI can also affect the proform 
A

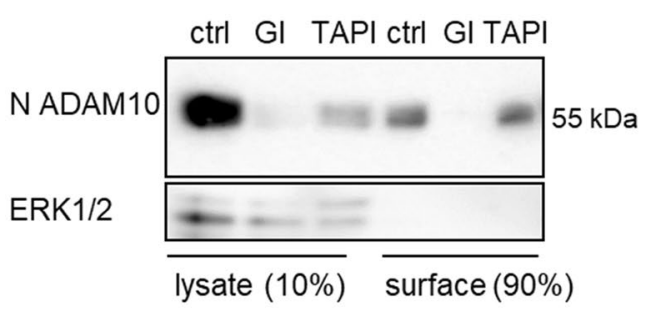

B

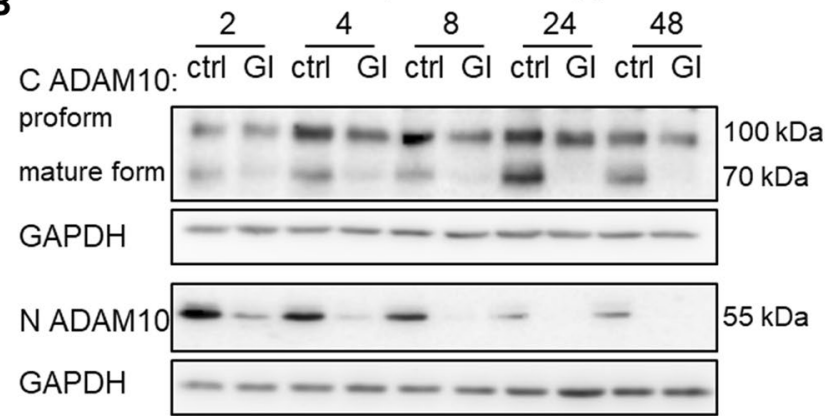

C

\section{$2 \mathrm{~h}$ pretreatment}

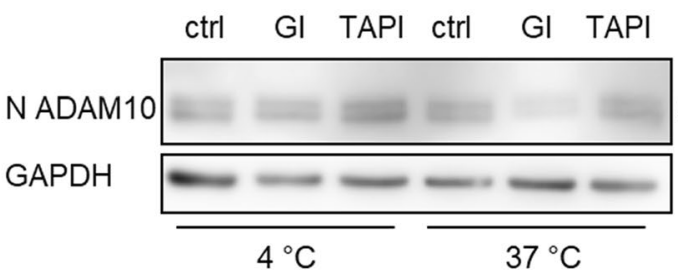

D

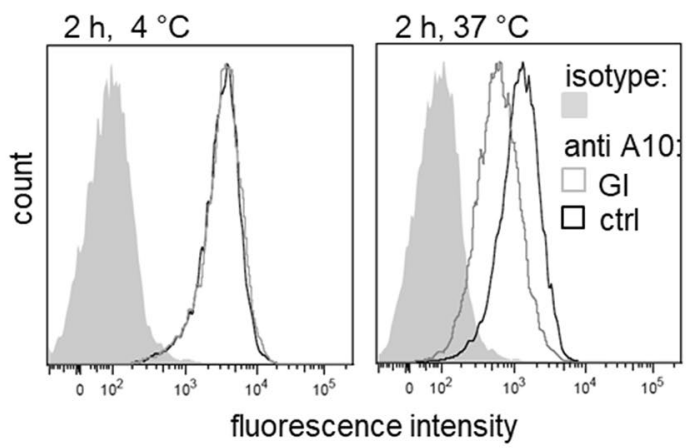

Fig. 2 Effect of GI on ADAM10 in surface precipitates and cell lysates and temperature dependence of ADAM10 downregulation. a THP- 1 cells were treated with $10 \mu \mathrm{M}$ GI, $10 \mu \mathrm{M}$ TAPI or vehicle control for $4 \mathrm{~h}$. Surface proteins were biotinylated on intact cells and subsequently precipitated from cell lysates. Precipitates and lysates were then probed by western blotting with an antibody against the $\mathrm{N}$-terminus of ADAM10. Detection of total cytosolic ERK1/2 served as a control. b THP-1 cells were treated with $10 \mu \mathrm{M}$ GI or vehicle control for the indicated periods of time. Cells were lysed and lysates were then probed by western blotting with antibodies against the $\mathrm{N}$-terminus or C-terminus of ADAM10 or against GAPDH as loading control. c THP- 1 cells were treated with $10 \mu \mathrm{M}$ GI, $10 \mu \mathrm{M}$ TAPI or vehicle control for $2 \mathrm{~h}$ at either $4{ }^{\circ} \mathrm{C}$ or $37^{\circ} \mathrm{C}$. Cells were cooled

of ADAM10 since the N-terminal antibody only detected mature ADAM10.

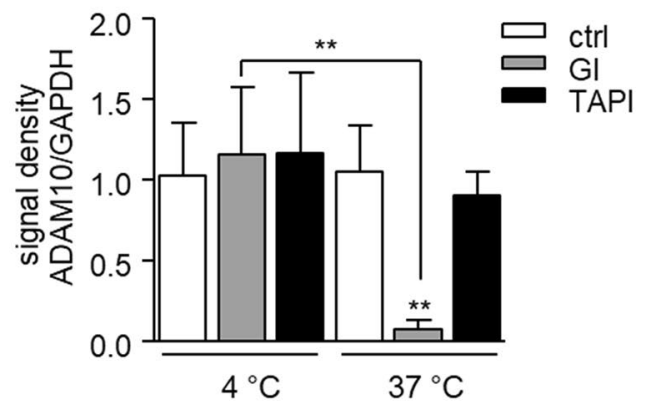

E

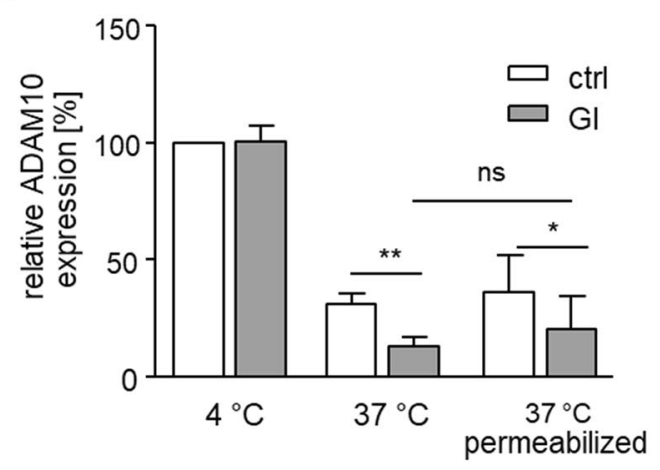

on ice, lysed, and probed by western blotting with antibodies against the N-terminus of ADAM10 or against GAPDH as loading control. Data are shown as representative western blot and as relative changes of band intensity determined by densitometric analysis. a-c One representative western blot out of three independent experiments. d-e THP-1 cells were first stained with primary antibody against ADAM10, then treated with $10 \mu \mathrm{M}$ GI or vehicle control for $2 \mathrm{~h}$ at either $4{ }^{\circ} \mathrm{C}$ or $37{ }^{\circ} \mathrm{C}$ and afterwards incubated with the secondary antibody on ice. Cells were either left intact or permeabilized before detection of ADAM10 by flow cytometry. Data were obtained in five independent experiments and are shown as representative histogram (d) or as means and SD of the geometric mean fluorescence in relation to that of the $4{ }^{\circ} \mathrm{C}$ control (e)

\section{GI254023X does not downregulate the proform of ADAM10}

To provide further evidence for downregulation of ADAM10, we used an antibody against the intracellular 
A

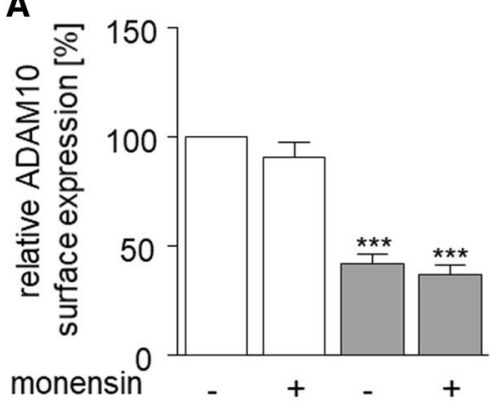

C

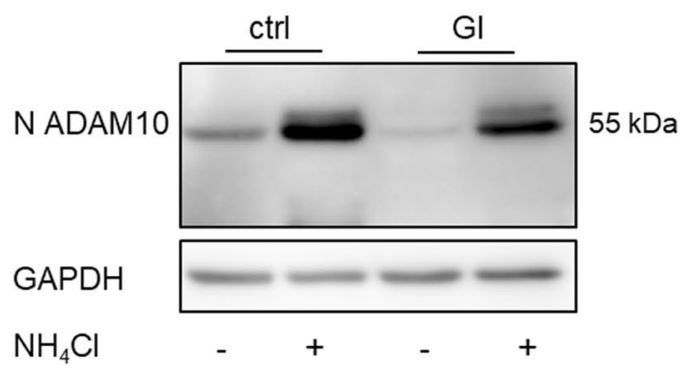

D

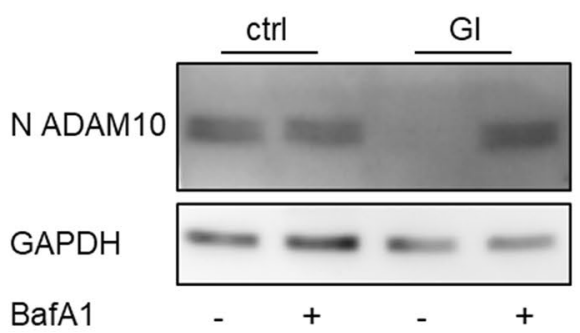

E

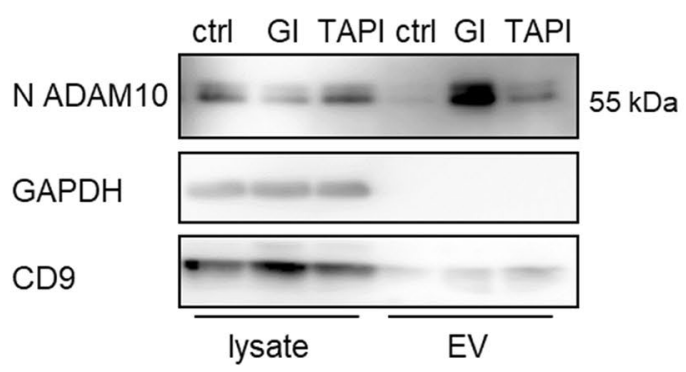

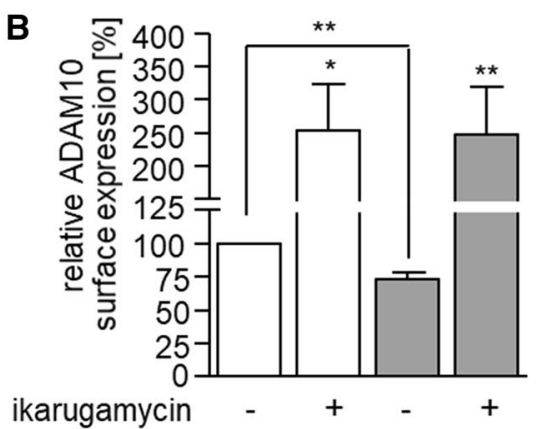
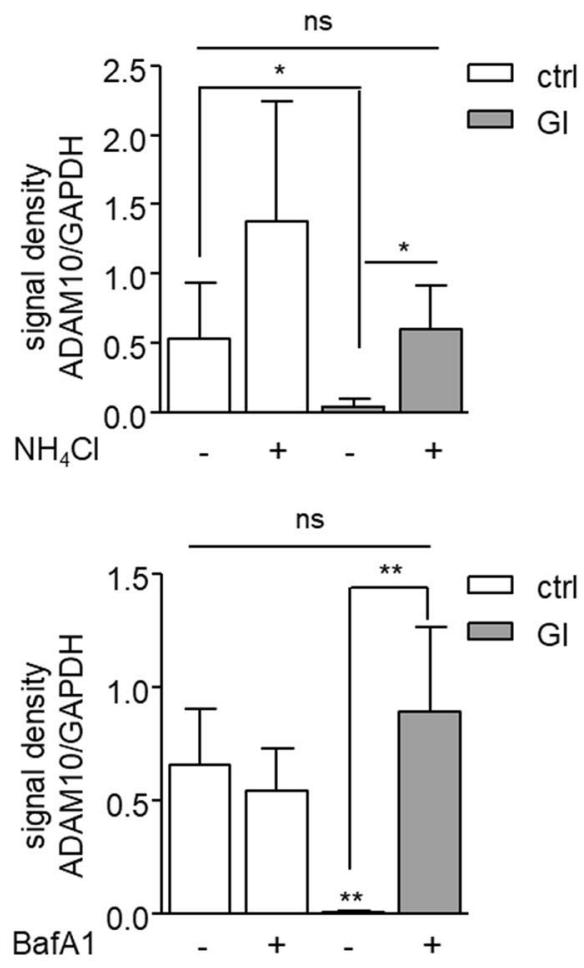

F
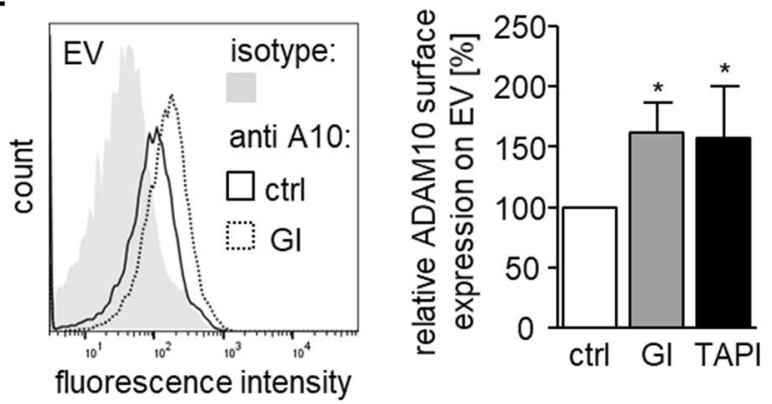

studies [21, 23]. The specificity of this detection was again shown by shRNA-mediated knockdown of ADAM10 resulting in complete loss of both protein bands (suppl. Fig. 2A).

It was shown recently that GI prevents C-terminal autodegradation of ADAM10 as an artefact of cell lysis [34]. Therefore, we always added GI to our lysis buffer which is especially needed when using the C-terminal ADAM10 
4Fig. 3 Effect of metalloproteinase inhibitors on ADAM10 internalization, degradation, and release in microvesicles. a, b THP-1 cells were treated with $10 \mu \mathrm{M}$ GI or vehicle control. After $4 \mathrm{~h}$ (a) or $2 \mathrm{~h}$ (b), cells were analyzed for surface expression of ADAM10 by flow cytometry. The geometric mean fluorescence of treated cells was calculated in relation to that of the respective control and summarized as mean and SD of three independent experiments.) THP-1 cells were treated with $10 \mu \mathrm{M}$ GI or vehicle control for $16 \mathrm{~h}$ in the absence or presence of $40 \mathrm{mM} \mathrm{NH} 4 \mathrm{Cl}$ (c) or $0.5 \mu \mathrm{M}$ bafilomycin $\mathrm{A} 1$ (d). Cell lysates were probed by western blotting with antibodies against the $\mathrm{N}$-terminus of ADAM10 or against GAPDH as loading control. Data are shown as representative western blot and as relative changes of band intensity determined by densitometric analysis of three independent experiments. e-f THP- 1 cells were treated with $10 \mu \mathrm{M} \mathrm{GI}$, $10 \mu \mathrm{M}$ TAPI or vehicle control for $24 \mathrm{~h}$. Extracellular vesicles (EV) were prepared from conditioned cell media by differential centrifugation. Lysates and EV preparation were then subjected to western blot analysis with antibodies against the N-terminus of ADAM10, against the exosomal marker CD9 or against GAPDH as internal control (e). EV preparations from conditioned media were conjugated to beads which were then studied for ADAM10 immunoreactivity by flow cytometry (f). Data are shown as representative result or as means and SD of four independent experiments

antibody. Consistent with previous reports, surface biotinylation showed that the pool of surface proteins contained both the pro- and the mature form of ADAM10 but only the mature form of ADAM17 (suppl. Fig. 2G) [13, 31, 35]. Both the $\mathrm{C}$ - and the N-terminal antibody detected the same time-dependent decrease of mature ADAM10 in the lysates after GI pretreatment (Fig. 2b, suppl. Fig. 2EF). Moreover, the proform of ADAM10 that could only be detected by the C-terminal antibody was not affected by GI. In summary, it can be concluded from our experiments that GI pretreatment of intact cells results in a loss of total mature ADAM10 but not the inactive proform.

\section{GI-induced ADAM10 downregulation is temperature dependent}

We then asked whether the loss of mature ADAM10 would be temperature dependent. For this, cells were exposed to GI at 4 and $37{ }^{\circ} \mathrm{C}$, lysed and studied by western blotting with the antibody against the N-terminus of ADAM10. We clearly noted that GI treatment only caused a loss of mature ADAM10 when the pretreatment was performed at $37{ }^{\circ} \mathrm{C}$ but not at $4{ }^{\circ} \mathrm{C}$ (Fig. 2c). These data suggest that GI-induced downregulation of mature ADAM10 is indeed temperature dependent.

We then studied GI-induced downregulation of surfaceexpressed ADAM10 on intact THP-1 cells by flow cytometry. Here, cells were incubated with the primary antibody against the $\mathrm{N}$-terminus of ADAM 10 at $4{ }^{\circ} \mathrm{C}$ prior to $\mathrm{GI}$ treatment. When subsequent GI treatment was performed at $4{ }^{\circ} \mathrm{C}$, no effect on the ADAM10 surface level was observed (Fig. 2d, e, suppl. Fig. 2H). Compared to the $4{ }^{\circ} \mathrm{C}$ treatment, we noted a general reduction of surface-expressed ADAM10 even in the absence of GI when cells were preincubated at $37{ }^{\circ} \mathrm{C}$ and this reduction was clearly more prominent when GI was present. When THP-1 cells were permeabilized to make intracellular ADAM10 available, the reduction of the ADAM10 signal after GI treatment at $37{ }^{\circ} \mathrm{C}$ was still significant (Fig. 2e). As a positive control, we used the antibody against the intracellular C-terminus of ADAM10. As expected, permeabilization of the cells increased the fluorescence signal when cells were incubated with this antibody (suppl. Fig. 2I). Of note, when comparing non-permeabilized with permeabilized cells after GI treatment, we did not observe a prominent increase in ADAM10 which could have resulted from accumulation of internalized protease after GI treatment (Fig. 2e).

\section{GI-induced ADAM10 downregulation coincides with ADAM10 release in extracellular vesicles}

We speculated that after GI treatment, ADAM10 may become internalized and subsequently degraded. When cells were treated with monensin which blocks protein forward trafficking, ADAM10 surface expression remained unaffected. However, additional GI treatment still induced downregulation of ADAM10 (Fig. 3a). Thus, an altered trafficking of ADAM10 from the Golgi to the cell surface appears unlikely. By contrast, the clathrin-mediated endocytosis inhibitor ikarugamycin caused a general increase of ADAM10 surface expression and prevented the GI-induced downregulation of ADAM10 from the cell surface (Fig. 3b). This finding confirms previous reports that ADAM10 undergoes constitutive internalization $[13,36]$ and also indicates that GI promotes internalization of ADAM10.

To study the potential involvement of lysosomal degradation, we treated cells with ammonium chloride $\left(\mathrm{NH}_{4} \mathrm{Cl}\right)$ or bafilomycin A1 which both block lysosomal acidification [37, 38]. Whereas bafilomycin A1 did not change the ADAM10 amount in the control cells, $\mathrm{NH}_{4} \mathrm{Cl}$ treatment clearly led to accumulation of ADAM10 in the lysates. Still, the surface expression of ADAM10 stayed unaltered under both agents (Fig. 3c, d, suppl. Fig. 3AB). Notably, ammonium chloride treatment partly rescued detection of ADAM10 in GI-treated cells which was even more pronounced when bafilomycin A1 was used. This indicates that the almost complete loss of ADAM10 due to GI treatment can be in part prevented by blocking lysosomal acidification.

Additionally, ADAM10 may be released in extracellular vesicles as shown in several previous studies [31, 39]. To investigate this possibility, we purified extracellular vesicles from THP-1 cells after GI or TAPI-1 treatment. In fact, loss of ADAM10 in the lysates correlated with a clear accumulation of ADAM10 in extracellular vesicles as detected by western blotting with either the N-terminal (Fig. 3e, suppl. 
Fig. 3C) or the C-terminal antibody (suppl. Fig. 3D). This increased release of vesicular ADAM10 was prominent with GI and much weaker with TAPI-1. To confirm these findings, isolated vesicles were coupled with beads which were then subjected to flow cytometry. Again, we observed significantly increased presence of ADAM10 on vesicles of those cells that were treated with the inhibitors (Fig. 3f).

\section{Recovery from GI-induced ADAM10 downregulation requires de novo synthesis}

To investigate recovery from GI-induced ADAM10 downregulation, the inhibitor was removed by extensive washing followed by incubation of the cells in the absence of the inhibitor. While ADAM10 expression was profoundly reduced directly after inhibitor treatment, it was partly restored after $8 \mathrm{~h}$ and fully restored after $24 \mathrm{~h}$ of recovery from inhibitor treatment. This effect was seen for ADAM10 surface expression by flow cytometry as well as for total cellular ADAM10 expression by western blotting, whereas the transcriptional level of ADAM10 stayed unaltered (suppl. Fig. 4A-C).

In the next experiment, part of the samples received monensin during the recovery period to prevent transport of ADAM10 from the Golgi network through the secretory pathway to the cell membrane. Monensin treatment clearly
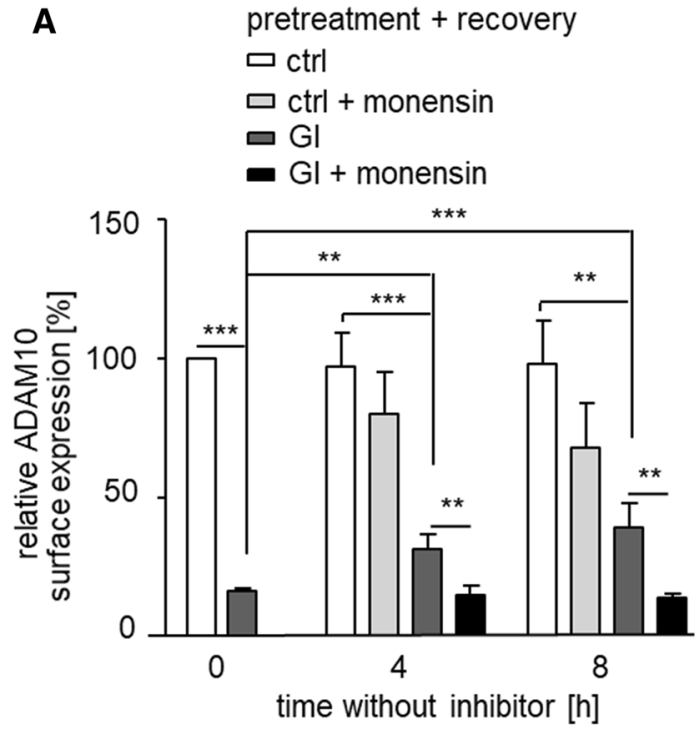

C

$24 \mathrm{~h}$ pretreatment

ctrl GI TAPI ctrl GI TAPI ctrl GI TAPI

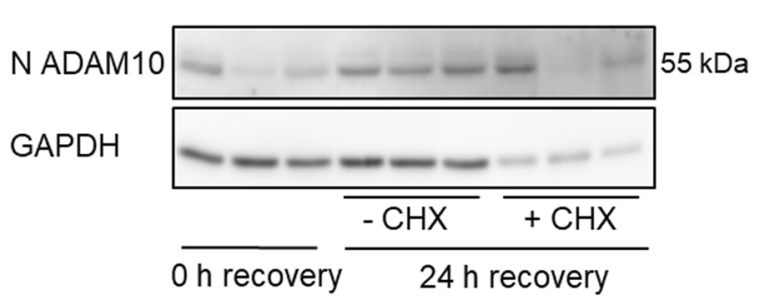

Fig. 4 Recovery from ADAM10 depletion by de novo synthesis. a THP-1 cells were treated with $10 \mu \mathrm{M}$ GI or vehicle control for $24 \mathrm{~h}$. After washing, $2 \mu \mathrm{g} / \mathrm{ml}$ monensin or vehicle, control was added and cells were incubated for the indicated periods of recovery time before they were analyzed for surface expression of ADAM10 by flow cytometry. b THP-1 cells were treated with $10 \mu \mathrm{M}$ GI or vehicle control for $24 \mathrm{~h}$. After washing, $100 \mu \mathrm{M}$ cycloheximide (CHX) or vehicle control was added and cells were incubated for the indicated periods of recovery time before they were analyzed for ADAM10 surface expression. ${ }^{* *} p<0.001$ compared with ctrl at the respective time
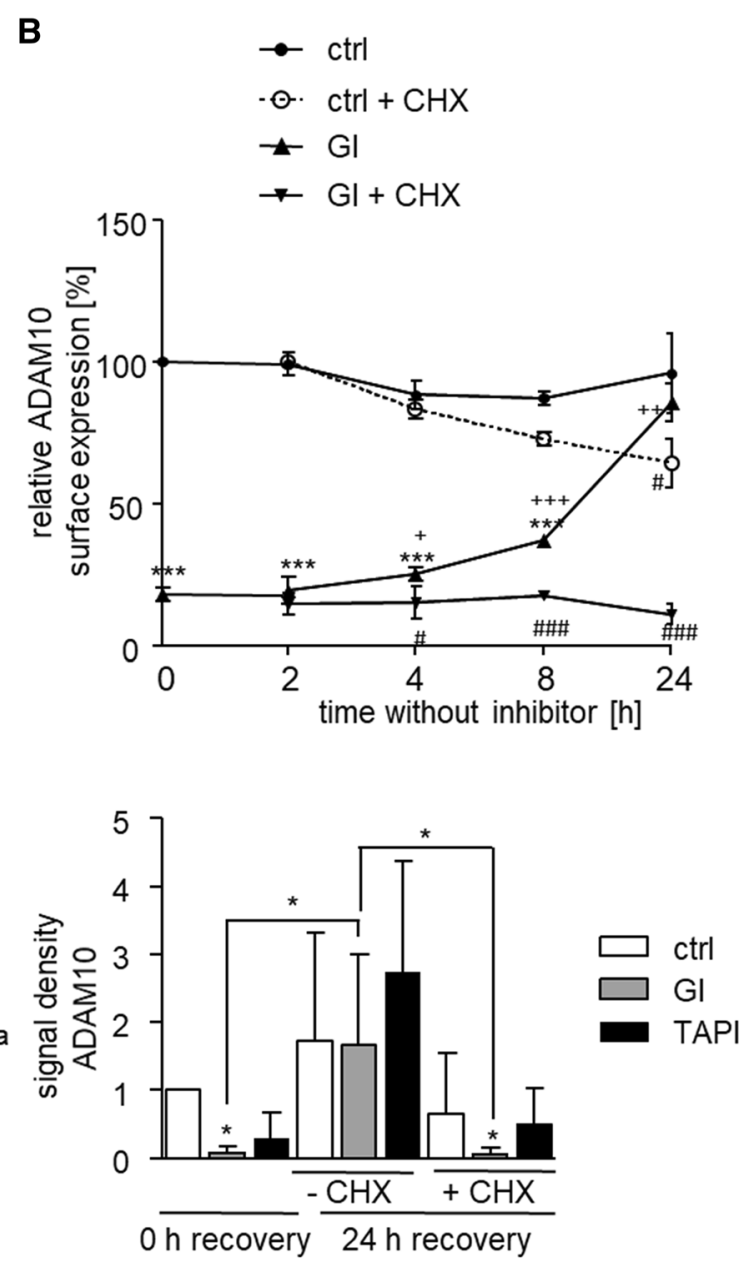

point, ${ }^{\#} p<0.05$ comparison betwee $n \pm \mathrm{CHX},{ }^{+} p<0.05$ compared with 0 h GI. c THP- 1 cells were treated with $10 \mu \mathrm{M}$ GI, $10 \mu \mathrm{M}$ TAPI or vehicle control for $24 \mathrm{~h}$. Washed cells were either further incubated with $100 \mu \mathrm{M}$ cycloheximide (CHX) or vehicle control for $24 \mathrm{~h}$ to allow recovery or not further incubated. Subsequently, cells were lysed for western blot analysis with an antibody against the N-terminus of ADAM10 or against GAPDH as loading control. Relative changes of band intensity were determined by densitometric analysis. Data are shown as means and SD or as representative western blot of three independent experiments 
prevented the recovery of ADAM10 surface expression from GI treatment (Fig. 4a). By contrast, monensin had no significant effect on ADAM10 expression of control cells not treated with GI.

In a similar experiment, cycloheximide was used to block de novo synthesis of ADAM10. In the absence of cycloheximide, we observed full restoration of ADAM10 surface expression after $24 \mathrm{~h}$ of recovery from GI treatment (Fig. 4b). This recovery was completely prevented by cycloheximide. Worth mentioning, $24 \mathrm{~h}$ cycloheximide treatment resulted in a reduction of the ADAM10 levels also in the control cells, showing the inhibition of the normal continual de novo synthesis of ADAM10. These findings were confirmed by western blot analysis of ADAM10 expression in cell lysates with the N-terminal antibody (Fig. 4c).

\section{Downregulation of ADAM10 is associated with impaired cellular shedding activity}

We questioned whether the observed downregulation and restoration of ADAM10 expression after inhibitor treatment would have implications for ADAM10-mediated shedding events. As a substrate we chose CXCL16 that can be induced in THP- 1 cells by co-culture with bacteria and is shed at least in part via ADAM10 [23]. In fact, after GI treatment without recovery, we observed a strong reduction of CXCL16 shedding as determined by measurement of soluble CXCL16 in the culture supernatant (Fig. 5a). However, when the cells were allowed to recover from GI treatment, the majority of CXCL16 shedding was restored. To confirm these findings for a different cell type and for another substrate, we performed a shedding assay for betacellulin with HEK293 cells. For this, AP-coupled betacellulin (BTC) was transfected into HEK293 cells and the fusion protein was detected in the lysates and supernatants by an AP-activity assay. As described before, ADAM10-mediated shedding of betacellulin can be induced by cell stimulation with ionomycin [32]. We confirmed that ionomycin-induced betacellulin shedding was almost completely abrogated when GI was present during the shedding assay (Fig. 5b). Next, we pretreated cells with GI and removed the inhibitor before the assay. GI pretreatment for only $10 \mathrm{~min}$ did not affect the shedding activity (Fig. 5c). A likely explanation of this result is that short-term binding of GI to ADAM10 is not sufficient to yield a long-lasting effect on ADAM10 activity since inhibition by GI is rapidly reversible when the inhibitor is removed. However, when the pretreatment was extended to $24 \mathrm{~h}$, the shedding activity was clearly reduced (Fig. 5c). Thus, after downregulation of ADAM10 as a result of long-term GI exposure, shedding is suppressed. Finally, 24-h pretreatment with GI plus 24-h recovery restored betacellulin shedding to approximately $75 \%$ of the control (Fig. 5d). As we showed above, downregulation of ADAM10 after long-term exposure to GI can be reversed after $24 \mathrm{~h}$ by de novo synthesis of ADAM 10 consequently leading to restoration of its shedding activity.

\section{Physiological relevance of ADAM10 downregulation}

We next used the metalloproteinase inhibitor TIMP-1 to study whether downregulation of ADAM10 can also occur with a natural inhibitor that is known to bind and inhibit ADAM10 [16, 21]. Recombinant human TIMP-1 was first tested for inhibition of ADAM10 activity in terms of betacellulin shedding. Pretreatment with a concentration of $200 \mathrm{nM}$ TIMP-1 for $30 \mathrm{~min}$ led to considerable inhibition of ADAM10 activity but not as prominent as the inhibition by GI (suppl. Fig. 5A). Furthermore, treatment with $200 \mathrm{nM}$ TIMP- 1 for $24 \mathrm{~h}$ clearly reduced the presence of mature ADAM10 in cell lysates (Fig. 6a and suppl. Fig. 5B). Again, this effect was not as fast and as strong as that of GI. Finally, we questioned whether a high concentration of a described peptide substrate that binds to the active site of ADAM10 would cause oversaturation of the protease and thereby result in a similar downregulation of ADAM10. In fact, 24-h treatment with an optimized peptide [40] for ADAM10-mediated cleavage could diminish ADAM10 surface expression (Fig. 6b). These data clearly indicate that natural TIMP-1 and also high amounts of soluble substrate molecules can cause depletion of ADAM10. Thus, downregulation of ADAM10 is not restricted to synthetic inhibitors but represents a physiological phenomenon occurring with endogenous inhibitors and potentially also with high concentrations of soluble substrates.

Consequently, the next question we asked was whether GI-mediated downregulation of ADAM10 would be limited to human cells or also occur in mice. For this, we started experiments with the murine macrophage cell line RAW264.7 which showed GI-induced downregulation of ADAM10 after 24 to a comparable extent as seen for human cells (suppl. Fig. 5CD). Next, we wanted to test the GI effect in an ex vivo setting. Therefore, murine bone marrow-derived macrophages (BMDMs) were generated from femur and tibia (suppl. Fig. 5E) and murine leukocytes were prepared from retro-orbital blood samples of C57BL/6 mice. In both cases, GI treatment led to a clear downregulation of ADAM10 (Fig. 6c, d, suppl. Fig. 5F).

Having found that GI reduces ADAM10 in primary murine cells, we finally wanted to see if this effect is also present in vivo. Therefore, mice were treated with GI by repeated i.p. injection. As demonstrated by flow cytometry, ADAM10 surface expression was clearly reduced in murine 

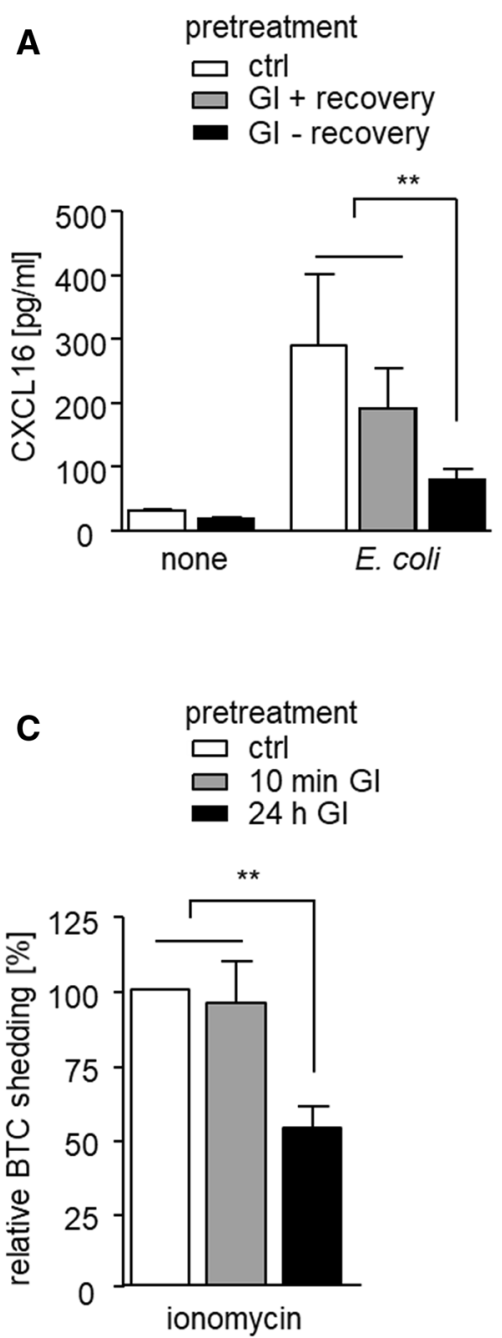

Fig. 5 Loss and recovery of shedding activity after ADAM10 depletion by GI. a THP-1 cells were incubated with $10 \mu \mathrm{M}$ GI or vehicle control and treated with pHrodo E. coli particles to induce CXCL16 expression or left untreated for $24 \mathrm{~h}$. After washing, cells were incubated for another $24 \mathrm{~h}$ with pHrodo $E$. coli particles in the presence or absence of inhibitor and released CXCL16 was quantified in the conditioned media by ELISA. b HEK293 cells transfected with a betacellulin (BTC) alkaline phosphatase (AP) fusion protein were pretreated with $10 \mu \mathrm{M}$ GI or vehicle control for $30 \mathrm{~min}$ and then stimulated with $1 \mu \mathrm{M}$ ionomycin to increase ADAM10 activity or left unstimulated. After $45 \mathrm{~min}$, the ratio of released to cell expressed BTC was

leukocytes derived from peripheral blood and isolated lung and liver cells (Fig. 6e). Furthermore, the total cellular amount of ADAM10 was also decreased in the liver and lung cells (Fig. 6f, suppl. Fig. 5G).

We then asked whether this ADAM10 downregulation occurs as a result of ADAM10 being inactive. To further investigate this, we introduced a single $\mathrm{E}$ to $\mathrm{A}$ amino acid residue replacement (E385A) at the active site of murine ADAM10 which is known to block the ADAM10 catalytic mechanism [41]. In another mutant, we switched the
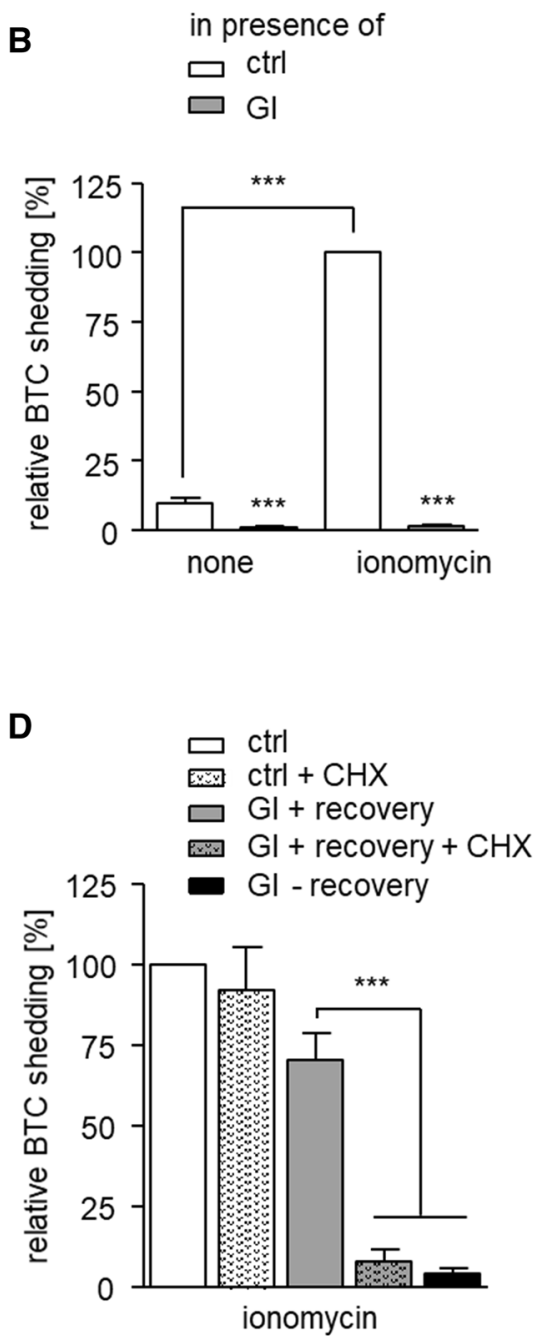

determined by means of an AP activity assay. c BTC-AP transfected HEK293 cells were incubated with $10 \mu \mathrm{M}$ GI or vehicle control for $10 \mathrm{~min}$ or $24 \mathrm{~h}$. The inhibitor was removed by washing and cells were studied for ionomycin-induced BTC release as in b. d BTC-AP transfected HEK293 cells were incubated with $10 \mu \mathrm{M}$ GI or vehicle control for $24 \mathrm{~h}$. Cells were washed and either further incubated for $24 \mathrm{~h}$ to recover from inhibitor treatment or not allowed to recover (CHX treatment), before they were studied for ionomycin-induced BTC shedding as in $\mathbf{b}$. Data in $\mathbf{b}-\mathbf{d}$ were normalized to the ionomycin treated control. Data are shown as means and SD of three independent experiments

positions of $\mathrm{H}(\mathrm{H} 394 \mathrm{E})$ and $\mathrm{E}(\mathrm{E} 385 \mathrm{H})$ in the zinc-binding motif to disrupt the catalytic mechanism without changing the amino acid residue composition and the charges. Switching the glutamate residue with one of the histidine residues was earlier shown to abrogate protease activity of the structural-related metzincins MMP9 and ADAM17 without disrupting the structural integrity of their catalytic domain since interaction with TIMP-1 or TIMP-3, respectively, was not altered [42, 43]. Modeling of the introduced amino acid replacements showed that the E385A mutant lacked the 
glutamate for the catalytic mechanism and the zinc ion and water coordination may be affected, whereas in the H394E mutant, the zinc ion is displaced but its coordination with water should be unaffected [42, 43] (Fig. 7a). Importantly, these changes should not affect the substrate binding pockets or other parts of the metalloproteinase structure. These murine variants as well as murine wild-type ADAM10 were then expressed in a HEK293 cell line with knockout of endogenous ADAM10. Activity of wild-type ADAM10 and activity loss of the variants was confirmed by means of a betacellulin cleavage assay (suppl. Fig. 6A). Western blot analysis revealed that all variants were expressed at a comparable level with respect to their proforms. By contrast, the mature forms of the inactive variants were expressed at a much lower level compared to that of wild-type ADAM10 further demonstrating that inactivation of ADAM10 causes downregulation of its mature form (Fig. 7b). Moreover, GI treatment only reduced the expression of murine wild-type ADAM10, but did not further suppress the residual surface expression of the inactive variants (Fig. 7c). Nevertheless, at this stage, it could not be excluded that the mutants differ in their ability to bind the small molecule inhibitor which then might have led to differences in downregulation of the protease variants. We, therefore, used a Cy5.5-labelled derivate of the small molecule inhibitor MN8 against ADAM10. The inhibitor caused similar ADAM10 downregulation as GI254023X (compare suppl. Fig. 1E) and via its fluorescence, it also allows to investigate binding of the inhibitor to ADAM10. In fact, flow cytometric experiments indicated that the relative binding of the small molecule inhibitor was not compromised by introducing the mutations (Fig. 7d, suppl. Fig. 6BC). Thus, the observed differences in GIinduced downregulation of mature ADAM10 between the mutants and wild-type ADAM10 (Fig. 7c) were not due to differences in their inhibitor binding but rather differences in their proteolytic activity.

Taken together, these findings indicate that ADAM10 activity is required to maintain the full level of ADAM10 on the cell surface and that the observed downregulation could represent a physiological mechanism to remove the inactive protease.

\section{Discussion}

In vitro and in vivo studies suggest that ADAM10 inhibition can suppress inflammatory and proliferative processes by shedding of inflammatory mediators or growth factors from the cell surface $[1,2,44]$. We here provide multiple lines of evidence that ADAM10 inhibitors not only block the active site of ADAM10 but also induce its downregulation. While inhibition of the active site is a rapid process occurring within minutes, we observed that downregulation of ADAM10 is temperature dependent and rather slow, requiring hours. This depletion is associated in parts with ADAM10 internalization and subsequent lysosomal degradation as well as with release of ADAM10 in extracellular vesicles. Full recovery from this depletion of ADAM10 takes several hours and requires de novo synthesis of ADAM10. Surface depletion of ADAM10 reduces shedding of the ADAM10 substrates CXCL16 and betacellulin. Consistent with the requirement of de novo synthesis after GI-mediated ADAM10 depletion, the recovery of the shedding activity is slow. We show that depletion of ADAM10 is not only an effect of synthetic inhibitors but also a physiological phenomenon also occurring with the natural inhibitor TIMP-1 and with overstraining the enzyme activity by oversaturating the protease with high amounts of substrate peptides. Moreover, inhibitor-mediated downregulation of ADAM10 can also be observed in vivo. Finally, downregulation is only observed with active ADAM10 but not with proteolytically inactive variants.

We found that long-term GI treatment of cells leads to downregulation of mature ADAM10 from the cell surface. This was evidenced by surface analysis of ADAM10 via flow cytometry as well as by surface precipitation of ADAM10 and western blot detection with an antibody against the N-terminus of ADAM10. Since the latter detection procedure was performed under denaturing conditions, it is very unlikely that GI remains bound to the protease and prevents the detection. In fact, GI not only reduces cell surface-expressed ADAM10 but also total mature ADAM10 as evidenced by western blotting with $\mathrm{N}$ - or C-terminustargeting antibodies. We further show that this loss of ADAM10 occurs in many different cell types and is not seen for ADAM17 suggesting that it is truly an effect of the specific interaction of ADAM10 and its inhibitor.

The binding of either small synthetic inhibitors, the natural inhibitor TIMP-1 to the active site of ADAM10 or the oversaturation of the protease with substrate peptides can sufficiently induce downregulation of the protease. Since TIMP-1 is a large protein, it may cause surface downregulation of ADAM10 through a temperature-dependent mechanism, similar to that of an antibody when binding to a surface antigen. However, our results obtained with small molecule inhibitors or peptides, as well as the experiments with the inactive variants of ADAM10 strongly suggest that the inhibition of the shedding activity is responsible for the downregulation of the protease. When substrate cleavage is hindered by the inhibitors or by mutation of the catalytic site, the protease might remain associated with its substrate. This complex could then be removed possibly via regulatory mechanisms of the substrate. By such a mechanism, ADAM10 would be sorted together with its substrate for either internalization and subsequent recycling, lysosomal degradation or release in extracellular vesicles. This may 

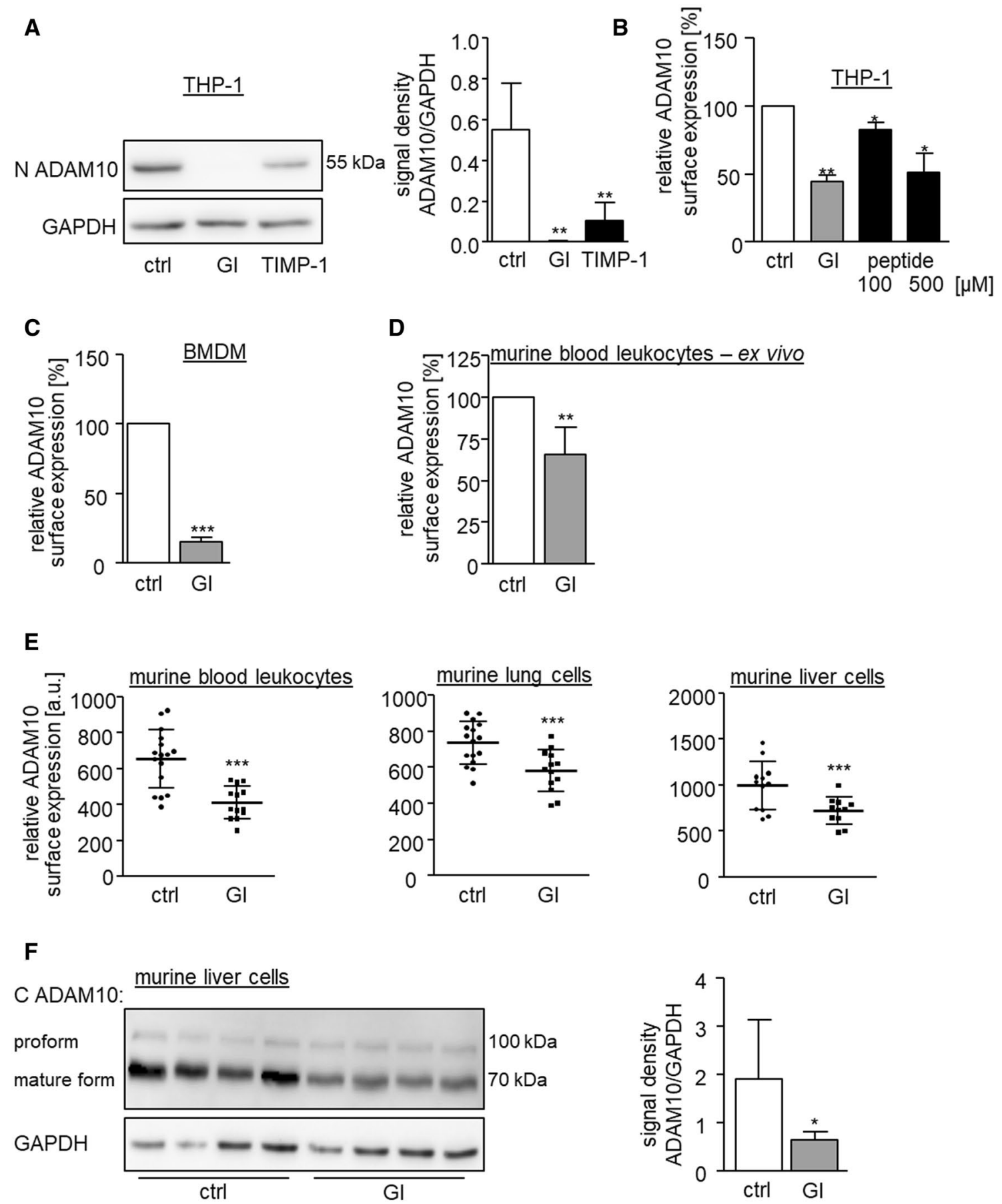

also involve adapter molecules that interact with ADAM10 and regulate its surface expression such as the TspanC8 subfamily of tetraspanins [12, 45]. For instance, Tspan15, Tspan5, and Tspan14 differentially control ADAM10-mediated cleavage of distinct substrates [46, 47]. Further analysis of GI-mediated effects on these tetraspanins may provide new mechanistic insight into the natural regulatory mechanisms of ADAM10 surface expression and activity.

GI-induced ADAM10 downregulation only occurs at $37{ }^{\circ} \mathrm{C}$ indicating that cellular metabolism and function is required. In general, several mechanisms may account for temperature-dependent depletion of ADAM10. ADAM10 has been shown to be constitutively released in extracellular vesicles, while ADAM17 release occurs predominantly upon cell stimulation $[15,31]$. Inhibitor treatment causes increased release of ADAM10 in extracellular vesicles as demonstrated by flow cytometry and by western blotting of isolated vesicles. Consistent with the surface downregulation of only the mature ADAM10 form, the vesicular ADAM10 had a molecular size corresponding to that of the mature 
4Fig. 6 In vitro and in vivo relevance of ADAM10 downregulation. a THP- 1 cells were treated with $200 \mathrm{nM}$ recombinant TIMP-1, $10 \mu \mathrm{M}$ GI or vehicle control for $24 \mathrm{~h}$ and subsequently studied for expression of mature ADAM10 by western blot analysis of cell lysates with antibodies against the N-terminus of ADAM10 and GAPDH as loading control. Data are shown as representative western blot and as relative changes of band intensity determined by densitometric analysis of three independent experiments. b THP-1 cells were treated with $10 \mu \mathrm{M}$ GI, the indicated amounts of a specific ADAM10 peptide substrate or vehicle for $2 \mathrm{~h}$ and subsequently analyzed for surface expression of ADAM10 by flow cytometry. Geometric mean fluorescence intensity was calculated in relation to that of the untreated control $(n=3)$. c Isolated murine BMDMs were treated with $10 \mu \mathrm{M}$ GI or vehicle control for $24 \mathrm{~h}$ and subsequently analyzed for surface expression of ADAM10 by flow cytometry. $\mathbf{d}$ Isolated murine peripheral blood leukocytes were treated with $10 \mu \mathrm{M}$ GI, $10 \mu \mathrm{M}$ TAPI or vehicle control for $2 \mathrm{~h}$ and subsequently analyzed for surface expression of ADAM10 by flow cytometry. In $\mathbf{c}$, $\mathbf{d}$, the geometric mean fluorescence of the cells was calculated in relation to that of the control and summarized as mean and SD of seven (c) or five (d) independent experiments. e, f Mice received daily intraperitoneal injections of $200 \mathrm{mg} / \mathrm{kg}$ bodyweight GI or DMSO as vehicle control. After 13 days, animals were sacrificed and ADAM10 surface expression on isolated peripheral blood leukocytes, lung or liver cells (e) was studied by flow cytometry. Data are shown as geometric mean fluorescence $(n \geq 11)$. $\mathbf{f}$ The western blot analysis of lysed liver tissue of four GI-treated and four control-treated mice with an antibody against the C-terminus of ADAM10 and GAPDH as loading control and the relative changes of band intensity of mature ADAM10 determined by densitometric analysis

form. It is unclear if this release in extracellular vesicles happens by direct budding of vesicles from the cell surface or if ADAM10 is internalized and afterwards released in extracellular vesicles. Besides this release in extracellular vesicles, other mechanisms may also contribute to surface downregulation of ADAM10. ADAM10 was found to be cleaved from the cell surface by ADAM15 and ADAM9 [14]. However, this would lead to the generation of cleavage fragments and in our experimental setup, no fragments were detected. ADAM10 may also be internalized and stored in vesicles $[13,48]$. In fact, GI-induced downregulation of ADAM10 can be prevented with ikarugamcyin suggesting that ADAM10 undergoes clathrin-dependent internalization. Interestingly, we did not detect more ADAM10 when cells were permeabilized to make the intracellular pool of ADAM10 accessible. Moreover, our western blot analysis of the cell lysates demonstrates a clear loss of total mature ADAM10 upon GI treatment. This loss can be prevented by blocking lysosomal acidification with ammonium chloride or bafilomycin A1 indicating that internalized ADAM10 is degraded in the lysosomes.

Of note, the proform of ADAM10 which is thought be inactive is not regulated by GI although it can also be found at the cell surface [49]. Similarly, we found that the inactive ADAM10 variants can also be expressed at the cell surface and are insensitive to GI treatment. Different scenarios may be possible to explain these findings.
Similar to the inactive ADAM10 variants, the proform of ADAM10 is constitutively transported to the cell surface where it is constitutively downregulated due to missing activity. However, the kinetic of this process would have to be in such a manner, that detecting immature ADAM10 on the cell surface is possible. Another putative explanation is that the ADAM10 proform is stable on the cell surface regardless of its catalytic inactivity because the presence of the prodomain might prevent downregulation by, for instance, hindering the binding to substrates.

The synthetic inhibitor GI has been used in several studies to block the activity of ADAM10 in cell culture experiments [17]. The inhibitor has also been administered in vivo to block shedding events in animal models of disease. For example, GI can prevent lung inflammatory processes and fibrosis development in lungs [10]. Additionally, ADAM10 can serve as a receptor for the $S$. aureus alpha toxin and GI was shown to suppress this toxicity in a murine model of $S$. aureus lung infection [11]. These findings indicate that treatment with GI could represent a therapeutic option especially in inflammatory, fibrotic, or infectious lung diseases. So far, it has been thought that the mode of action of GI would be limited to its inhibition of ADAM10 proteolytic activity. Our study demonstrates that GI causes downregulation of ADAM10 not only in an in vitro setting but also in leukocytes from peripheral blood and from liver and lung cells in vivo. This depletion of surface-expressed ADAM10 would not only affect metalloproteinase-related functions but also binding functions of the protease such as potential interaction with integrins or with bacterial toxins. When GI is removed from the system, it can be expected that inhibition will still persist for some time since ADAM10 has to be resynthesized to restore surface expression. This has to be considered when ADAM10 inhibition is no longer wanted or treatment cannot be continued due to side effects. Nevertheless, some recovery from downregulation can be observed after $24 \mathrm{~h}$. In case further ADAM10 inhibition is necessary, it would be advisable to re-administer GI within this time frame.

Endogenous inhibitors such as TIMP-1 can control excess ADAM10 activity [16]. Our study shows that TIMP- 1 or even an excess of ADAM10 substrate peptides that are derivatives of the regions of natural substrates, which harbor the ADAM10 cleavage sites, can induce downregulation of ADAM10 similar as seen for GI. This indicates that the regulation of ADAM10 is not only limited to synthetic compounds but also represents a physiological phenomenon. Taken together, we propose that this downregulation represents a natural mechanism to remove ADAM10 from the cell surface in a situation when endogenous or exogenous molecules impact the activity of the protease. Concurrently, bound inhibitors would also be removed as they would either undergo degradation 
A

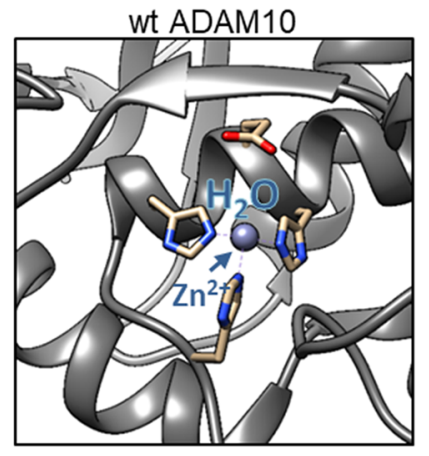

ADAM10 E385A

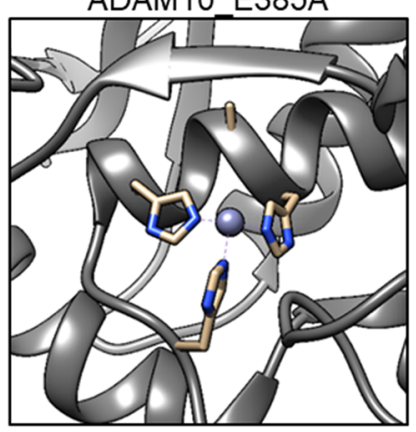

ADAM10 E385H H394E

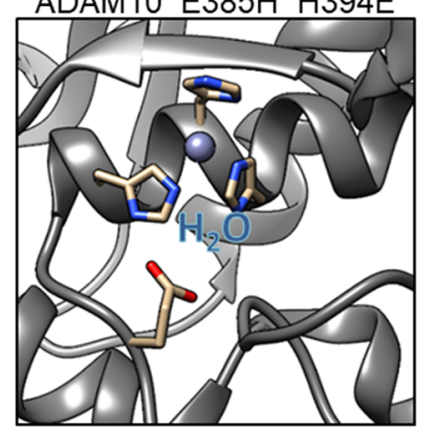

B

\section{ADAM10:}
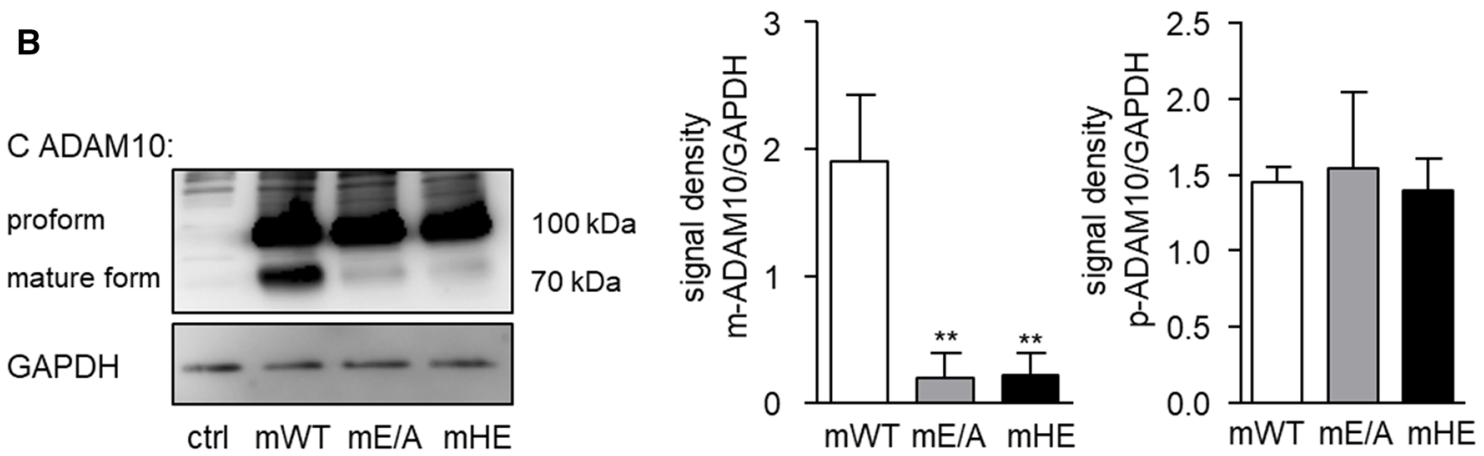

C

D
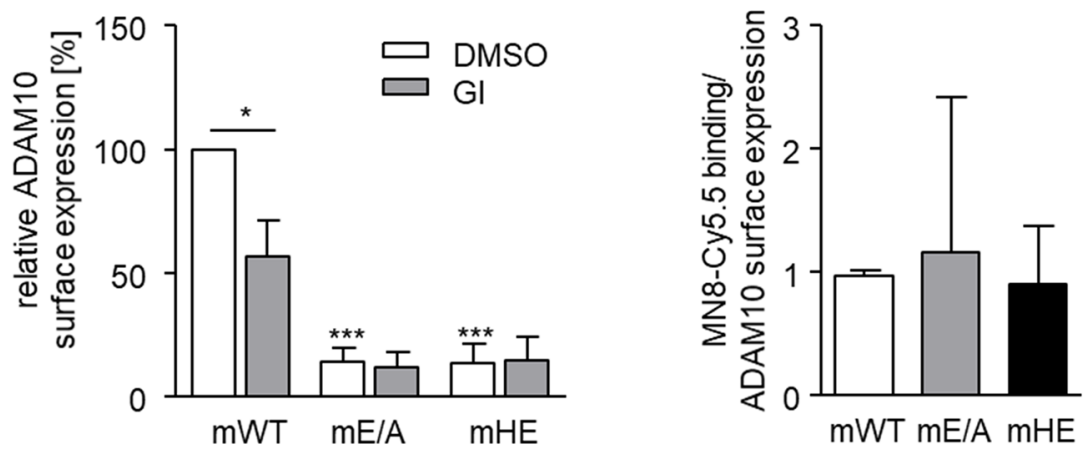

Fig. 7 Expression and regulation of wild-type and inactive murine ADAM10 variants. a The active site within the catalytic domain of wt ADAM10 is depicted based on the ADAM10 structure pdb: 6be6 [19]. Here the three shown histidine residues coordinate the zinc ion $\left(\mathrm{Zn}^{2+}\right)$, which together with the glutamate residue binds the water molecule needed for the hydrolysis of the peptide backbone of substrates. Therefore, substitution of the glutamate residue with an alanine residue (E385A) causes the loss of the protease activity. Changing the position of E385 with $\mathrm{H} 394$ results in a shifted active site which is not able to catalyse the hydrolysis of peptide bonds. b-d ADAM10-deficient HEK293 cells were transfected to express either murine WT-ADAM10 or the catalytically inactive murine ADAM10 mutants (mE/A-ADAM10 or mHE-ADAM10). Results are shown as representative western blot analysis of lysed HEK293 cells with an

antibody against the C-terminus of ADAM10 and GAPDH as loading control (b). Relative changes in band intensity of mature and proform of ADAM10 were determined by densitometric analysis of three independent experiments. c Transfected cells were treated with $20 \mu \mathrm{M}$ GI or vehicle control for $24 \mathrm{~h}$ and studied for murine ADAM10 surface expression. Results are shown as the geometric mean fluorescence of the ADAM10 expressing cells in relation to that of the control (murine WT-ADAM10 expressing cells) and summarized as mean and SD of three independent experiments. $\mathbf{d}$ Binding of Cy5.5-labeled MN8 to transfected HEK293 cells expressing ADAM10 variants was determined by flow cytometry. The specific fluorescence signal was calculated in relation to that of the ADAM10 surface expression determined for each variant $(n=3$, see suppl. Fig. 6) 
or exosomal release together with inactivated ADAM10 and this would then allow to restore ADAM10 activity over time by de novo synthesis of the protease. Furthermore, one may speculate that other cell-surface proteases undergo a similar regulation, which would introduce a new aspect to consider, when analyzing these proteases in search for ways to therapeutically block their activity.

Acknowledgements Open Access funding provided by Projekt DEAL. We thank T. Woopen for technical assistance. This work was supported in part by the IZKF Aachen and by the DFG grants Lu869/5-2, Lu869/8-1 and Dr1013/1-1. C.Z. Koo is supported by British Heart Foundation PhD Studentship FS/18/9/33388. We thank Björn Rabe (University of Kiel, Germany) for providing ADAM10-deficient HEK293 cells and Shigeki Higashiyama (Ehime University, Japan) for providing the BTC-AP construct.

Author contributions AS performed experiments and analyses and contributed to the design of the study. SD assisted with molecular analysis, interpretation, and design of the study. JW assisted with data analysis and statistic. CZK, MGT, EN, DC, and AR provided special reagents and/or helped in analyzing the experiments. EN generated and provided the MN8 and MN8-Cy5.5 (CAM36) inhibitor. DY contributed to the design of the study and supervision. AL provided the general concept, study design, and supervision. AS, SD, and AL wrote the paper. All authors read and approved the final manuscript.

\section{Compliance with ethical standards}

Conflict of interest The authors declare that they have no conflict of interest.

Ethical approval The authors declare that the experiments comply with the current law of the country in which they were performed.

Open Access This article is licensed under a Creative Commons Attribution 4.0 International License, which permits use, sharing, adaptation, distribution and reproduction in any medium or format, as long as you give appropriate credit to the original author(s) and the source, provide a link to the Creative Commons licence, and indicate if changes were made. The images or other third party material in this article are included in the article's Creative Commons licence, unless indicated otherwise in a credit line to the material. If material is not included in the article's Creative Commons licence and your intended use is not permitted by statutory regulation or exceeds the permitted use, you will need to obtain permission directly from the copyright holder. To view a copy of this licence, visit http://creativecommons.org/licenses/by/4.0/.

\section{References}

1. Dreymueller D, Uhlig S, Ludwig A (2015) Adam-family metalloproteinases in lung inflammation: potential therapeutic targets. Am J Physiol Lung Cell Mol Physiol 2015:1

2. Pruessmeyer J, Ludwig A (2009) The good, the bad and the ugly substrates for ADAM10 and ADAM17 in brain pathology, inflammation and cancer. Semin Cell Dev Biol 1:1

3. Saftig P, Lichtenthaler SF (2015) The alpha secretase ADAM10: A metalloprotease with multiple functions in the brain. Prog Neurobiol 2015:1
4. Jorissen E, Prox J, Bernreuther C et al (2010) The disintegrin/ metalloproteinase ADAM10 is essential for the establishment of the brain cortex. J Neurosci. https://doi.org/10.1523/JNEUR OSCI.5221-09.2010

5. Weber S, Niessen MT, Prox J et al (2011) The disintegrin/metalloproteinase Adam 10 is essential for epidermal integrity and Notch-mediated signaling. Development. https://doi.org/10.1242/ dev.055210

6. Alabi RO, Glomski K, Haxaire C et al (2016) ADAM10-dependent signaling through Notch1 and Notch4 controls development of organ-specific vascular beds. Circ Res. https://doi.org/10.1161/ CIRCRESAHA.115.307738

7. Schulz B, Pruessmeyer J, Maretzky T et al (2008) ADAMIO regulates endothelial permeability and T-cell transmigration by proteolysis of vascular endothelial cadherin. Circ Res. https://doi. org/10.1161/CIRCRESAHA.107.169805

8. Hundhausen C, Schulte A, Schulz B et al (2007) Regulated shedding of transmembrane chemokines by the disintegrin and metalloproteinase 10 facilitates detachment of adherent leukocytes. J Immunol. https://doi.org/10.4049/jimmunol.178.12.8064

9. Tanida S, Joh T, Itoh K et al (2004) The mechanism of cleavage of EGFR ligands induced by inflammatory cytokines in gastric cancer cells. Gastroenterology. https://doi.org/10.1053/j.gastr o.2004.05.017

10. Lagares D, Ghassemi-Kakroodi P, Tremblay C et al (2017) ADAM10-mediated ephrin-B2 shedding promotes myofibroblast activation and organ fibrosis. Nat Med. https://doi. org/10.1038/nm.4419

11. Inoshima I, Inoshima N, Wilke GA et al (2011) A Staphylococcus aureus pore-forming toxin subverts the activity of ADAM10 to cause lethal infection in mice. Nat Med. https:// doi.org/10.1038/nm.2451

12. Matthews AL, Szyroka J, Collier R et al (2017) Scissor sisters: Regulation of ADAM10 by the TspanC8 tetraspanins. Biochem Soc Trans 2017:1

13. Carey RM, Blusztajn JK, Slack BE (2011) Surface expression and limited proteolysis of ADAM10 are increased by a dominant negative inhibitor of dynamin. BMC Cell Biol. https://doi. org/10.1186/1471-2121-12-20

14. Tousseyn T, Thathiah A, Jorissen E et al (2009) ADAM10, the rate-limiting protease of regulated intramembrane proteolysis of notch and other proteins, is processed by ADAMS-9, ADAMS15, and the $\gamma$-secretase. J Biol Chem. https://doi.org/10.1074/ jbc.M805894200

15. Stoeck A, Keller S, Riedle S et al (2006) A role for exosomes in the constitutive and stimulus-induced ectodomain cleavage of L1 and CD44. Biochem J. https://doi.org/10.1042/BJ20051013

16. Amour A, Knight CG, Webster A et al (2000) The in vitro activity of ADAM-10 is inhibited by TIMP-1 and TIMP-3. FEBS Lett. https://doi.org/10.1016/S0014-5793(00)01528-3

17. Dreymueller D, Ludwig A (2017) Considerations on inhibition approaches for proinflammatory functions of ADAM proteases. Platelets 2017:1

18. Ludwig A, Hundhausen C, Lambert M et al (2005) Metalloproteinase inhibitors for the disintegrin-like metalloproteinases ADAM10 and ADAM17 that differentially block constitutive and phorbol ester-inducible shedding of cell surface molecules. Comb Chem High Throughput Screen. https://doi. org/10.2174/1386207053258488

19. Seegar TCM, Killingsworth LB, Saha N et al (2017) Structural basis for regulated proteolysis by the $\alpha$-secretase ADAM10. Cell. https://doi.org/10.1016/j.cell.2017.11.014

20. Weskamp G, Ford JW, Sturgill J et al (2006) ADAM10 is a principal "sheddase" of the low-affinity immunoglobulin E receptor CD23. Nat Immunol. https://doi.org/10.1038/ni1399 
21. Hundhausen C, Misztela D, Berkhout TA et al (2003) The disintegrin-like metalloproteinase ADAM10 is involved in constitutive cleavage of CX3CL1 (fractalkine) and regulates CX3CL1mediated cell-cell adhesion. Blood. https://doi.org/10.1182/ blood-2002-12-3775

22. Matthews V, Schuster B, Schütze S et al (2003) Cellular cholesterol depletion triggers shedding of the human interleukin-6 receptor by ADAM10 and ADAM17 (TACE). J Biol Chem. https://doi.org/10.1074/jbc.M210584200

23. Abel S, Hundhausen C, Mentlein R et al (2004) The transmembrane CXC-chemokine ligand 16 is induced by IFN- $\gamma$ and TNF- $\alpha$ and shed by the activity of the disintegrin-like metalloproteinase ADAM10. J Immunol. https://doi.org/10.4049/jimmu nol.172.10.6362

24. Maretzky T, Reiss K, Ludwig A et al (2005) ADAM10 mediates E-cadherin shedding and regulates epithelial cell-cell adhesion, migration, and $\beta$-catenin translocation. Proc Natl Acad Sci USA. https://doi.org/10.1073/pnas.0500918102

25. Reiss K, Maretzky T, Ludwig A et al (2005) ADAM10 cleavage of $\mathrm{N}$-cadherin and regulation of cell-cell adhesion and $\beta$-catenin nuclear signalling. EMBO J. https://doi.org/10.1038/sj.emboj .7600548

26. Sanderson MP, Erickson SN, Gough PJ et al (2005) ADAM10 mediates ectodomain shedding of the betacellulin precursor activated by $p$-aminophenylmercuric acetate and extracellular calcium influx. J Biol Chem. https://doi.org/10.1074/jbc.M408804200

27. Camodeca C, Nuti E, Tosetti F, Poggi A, D'Arrigo C, Zocchi MR, Rossello A (2018) Synthesis and in vitro evaluation of ADAM10 and ADAM17 highly selective bioimaging probes. ChemMedChem 13(19):2119-2131. https://doi.org/10.1002/cmdc.20180 0482

28. Pruessmeyer J, Hess FM, Alert $\mathrm{H}$ et al (2014) Leukocytes require ADAM10 but not ADAM17 for their migration and inflammatory recruitment into the alveolar space. Blood. https://doi. org/10.1182/blood-2013-09-511543

29. Riethmueller S, Somasundaram P, Ehlers JC et al (2017) Proteolytic Origin of the soluble human IL-6R in vivo and a decisive role of N-glycosylation. PLoS Biol. https://doi.org/10.1371/journ al.pbio.2000080

30. Pruessmeyer J, Martin C, Hess FM et al (2010) A Disintegrin and metalloproteinase 17 (ADAM17) mediates inflammation-induced shedding of syndecan-1 and -4 by lung epithelial cells. J Biol Chem. https://doi.org/10.1074/jbc.M109.059394

31. Groth E, Pruessmeyer J, Babendreyer A et al (2016) Stimulated release and functional activity of surface expressed metalloproteinase ADAM17 in exosomes. Biochim Biophys Acta Mol Cell Res. https://doi.org/10.1016/j.bbamcr.2016.09.002

32. Sahin U, Weskamp G, Kelly K et al (2004) Distinct roles for ADAM10 and ADAM17 in ectodomain shedding of six EGFR ligands. J Cell Biol. https://doi.org/10.1083/jcb.200307137

33. Camodeca C, Nuti E, Tepshi L, Boero S, Tuccinardi T, Stura EA (2016) Discovery of a new selective inhibitor of a disintegrin and metalloprotease 10 (ADAM-10) able to reduce the shedding of NKG2D ligands in Hodgkin's lymphoma cell models. Eur J Med Chem 111:193-201

34. Brummer T, Pigoni M, Rossello A et al (2018) The metalloprotease ADAM10 (a disintegrin and metalloprotease 10) undergoes rapid, postlysis autocatalytic degradation. FASEB J. https://doi. org/10.1096/fj.201700823RR

35. Lorenzen I, Lokau J, Korpys Y et al (2016) Control of ADAM17 activity by regulation of its cellular localisation. Sci Rep. https:// doi.org/10.1038/srep35067
36. Eschenbrenner E, Jouannet S, Clay D et al (2020) TspanC8 tetraspanins differentially regulate ADAM10 endocytosis and half-life. Life Sci Alliance. https://doi.org/10.26508/lsa.201900444

37. Jurkovitz CT, England BK, Ebb RG, Mitch WE (1992) Influence of ammonia and $\mathrm{pH}$ on protein and amino acid metabolism in LLC-PK1 cells. Kidney Int. https://doi.org/10.1038/ki.1992.323

38. Yoshimori T, Yamamoto A, Moriyama Y et al (1991) Bafilomycin A1, a specific inhibitor of vacuolar-type H+-ATPase, inhibits acidification and protein degradation in lysosomes of cultured cells. J Biol Chem 1991:1

39. Gutwein P, Mechtersheimer S, Riedle S et al (2003) ADAM10mediated cleavage of $\mathrm{L} 1$ adhesion molecule at the cell surface and in released membrane vesicles. FASEB J. https://doi.org/10.1096/ fj.02-0430fje

40. Caescu CI, Jeschke GR, Turk BE (2009) Active-site determinants of substrate recognition by the metalloproteinases TACE and ADAM10. Biochem J. https://doi.org/10.1042/BJ20090549

41. Lammich S, Kojro E, Postina R et al (1999) Constitutive and regulated $\alpha$-secretase cleavage of Alzheimer's amyloid precursor protein by a disintegrin metalloprotease. Proc Natl Acad Sci USA. https://doi.org/10.1073/pnas.96.7.3922

42. Roderfeld M, Weiskirchen R, Wagner S et al (2006) Inhibition of hepatic fibrogenesis by matrix metalloproteinase- 9 mutants in mice. FASEB J. https://doi.org/10.1096/fj.05-4828com

43. Düsterhöft S, Höbel K, Oldefest $\mathrm{M}$ et al (2014) A disintegrin and metalloprotease 17 dynamic interaction sequence, the sweet tooth for the human interleukin 6 receptor. J Biol Chem. https://doi. org/10.1074/jbc.M114.557322

44. Saftig P, Reiss K (2011) The "A Disintegrin And Metalloproteases" ADAM10 and ADAM17: Novel drug targets with therapeutic potential? Eur J Cell Biol 2011:1

45. Saint-Pol J, Eschenbrenner E, Dornier E et al (2017) Regulation of the trafficking and the function of the metalloprotease ADAM10 by tetraspanins. Biochem Soc Trans 2017:1

46. Jouannet S, Saint-Pol J, Fernandez L et al (2016) TspanC8 tetraspanins differentially regulate the cleavage of ADAM10 substrates, Notch activation and ADAM10 membrane compartmentalization. Cell Mol Life Sci. https://doi.org/10.1007/s00018-015-2111-z

47. Noy PJ, Yang J, Reyat JS et al (2016) TspanC8 tetraspanins and A Disintegrin and Metalloprotease 10 (ADAM10) interact via their extracellular regions. J Biol Chem. https://doi.org/10.1074/jbc. m115.703058

48. Andrzejewski MG, Koelsch A, Kogel T et al (2010) Distinct role of the intracellular C-terminus for subcellular expression, shedding and function of the murine transmembrane chemokine CX3CL1. Biochem Biophys Res Commun. https:// doi.org/10.1016/j.bbrc.2010.03.139

49. Atapattu L, Saha N, Chheang C et al (2016) An activated form of ADAM10 is tumor selective and regulates cancer stem-like cells and tumor growth. J Exp Med. https://doi.org/10.1084/jem.20151 095

Publisher's Note Springer Nature remains neutral with regard to jurisdictional claims in published maps and institutional affiliations. 